\title{
Thrombin promotes diet-induced obesity through fibrin-driven inflammation
}

\author{
Anna K. Kopec, ${ }^{1}$ Sara R. Abrahams, ${ }^{2}$ Sherry Thornton, ${ }^{3}$ Joseph S. Palumbo, ${ }^{4}$ Eric S. Mullins, ${ }^{4}$ Senad Divanovic, ${ }^{5}$ Hartmut Weiler, ${ }^{6}$ \\ A. Phillip Owens III, ${ }^{7}$ Nigel Mackman, ${ }^{8}$ Ashley Goss, ${ }^{9}$ Joanne van Ryn, ${ }^{10}$ James P. Luyendyk, ${ }^{1}$ and Matthew J. Flick ${ }^{2}$ \\ 'Department of Pathobiology and Diagnostic Investigation, Institute for Integrative Toxicology, Michigan State University, East Lansing, Michigan, USA. ${ }^{2}$ Division of Experimental Hematology and Cancer \\ Biology, ${ }^{3}$ Division of Rheumatology, ${ }^{4}$ Division of Hematology, and ${ }^{5}$ Division of Immunobiology, Cincinnati Children's Hospital Research Foundation, Cincinnati, Ohio, USA. ${ }^{6}$ Department of Physiology, Blood \\ Center of Wisconsin, Milwaukee, Wisconsin, USA. 'Division of Cardiovascular Health and Disease, Department of Internal Medicine, University of Cincinnati College of Medicine, Cincinnati, Ohio, USA. \\ ${ }^{8}$ Thrombosis and Hemostasis Program, Division of Hematology and Oncology, Department of Medicine, UNC McAllister Heart Institute, University of North Carolina at Chapel Hill, Chapel Hill, North Carolina, \\ USA. ${ }^{9}$ Department of Cardiometabolic Disease Research, Boehringer Ingelheim Pharmaceuticals Inc., Ridgefield, Connecticut, USA. ${ }^{10}$ Department of Cardiometabolic Disease Research, Boehringer Ingelheim \\ Pharma GmbH, Biberach, Germany.
}

Obesity promotes a chronic inflammatory and hypercoagulable state that drives cardiovascular disease, type 2 diabetes, fatty liver disease, and several cancers. Elevated thrombin activity underlies obesity-linked thromboembolic events, but the mechanistic links between the thrombin/fibrin(ogen) axis and obesity-associated pathologies are incompletely understood. In this work, immunohistochemical studies identified extravascular fibrin deposits within white adipose tissue and liver as distinct features of mice fed a high-fat diet (HFD) as well as obese patients. Fib $\gamma^{390-396 A}$ mice carrying a mutant form of fibrinogen incapable of binding leukocyte $\alpha_{M} \beta_{2}$-integrin were protected from HFD-induced weight gain and elevated adiposity. Fib ${ }^{390-396 A}$ mice had markedly diminished systemic, adipose, and hepatic inflammation with reduced macrophage counts within white adipose tissue, as well as near-complete protection from development of fatty liver disease and glucose dysmetabolism. Homozygous thrombomodulin-mutant ThbdPro mice, which have elevated thrombin procoagulant function, gained more weight and developed exacerbated fatty liver disease when fed a HFD compared with WT mice. In contrast, treatment with dabigatran, a direct thrombin inhibitor, limited HFD-induced obesity development and suppressed progression of sequelae in mice with established obesity. Collectively, these data provide proof of concept that targeting thrombin or fibrin(ogen) may limit pathologies in obese patients.

\section{Introduction}

Obesity is a health care crisis of global pandemic status, with an estimated $34 \%$ of adults and $17 \%$ of children in the United States classified as obese as defined by a BMI greater than or equal to 30 $\mathrm{kg} / \mathrm{m}^{2}(1)$. The detrimental effects of obesity are due to the fact that it induces a state of chronic "metabolic inflammation" whereby the control of energy and metabolites in adipose, liver, and muscle tissue is directed largely by innate immune cells (e.g., macrophages) and soluble effector molecules (e.g., cytokines) (2-7). Metabolic inflammation predisposes individuals to metabolic syndrome and associated comorbidities, including cardiovascular disease, type 2 diabetes (T2D), nonalcoholic fatty liver disease (NAFLD), osteoarthritis, and various cancers that are the most frequent causes of death in the United States and most Westernized countries $(1,8)$.

Obesity is also characterized as a hypercoagulable state. Association studies have documented elevated circulating levels of von Willebrand factor, fibrinogen, factor VII, and biomarkers of coag-

Conflict of interest: A. Goss and J. van Ryn are employees of Boehringer Ingelheim Pharmaceuticals Inc.

Submitted: January 9, 2017; Accepted: June 2, 2017.

Reference information: / Clin Invest. 2017;127(8):3152-3166.

https://doi.org/10.1172/JCI92744. ulation activity (e.g., thrombin-antithrombin [TAT] complexes, thrombin $\mathrm{f1}+2$, D-dimer) in obese individuals (9-11). Activated protein $\mathrm{C}$ (APC) resistance increases with BMI, in part because of elevated factor VIII levels (12). A Mendelian randomization study determined that the risk of deep vein thrombosis increased 3.4fold for severely obese patients compared with normal-weight individuals (13). More notably, both initial risk and severe morbidity and mortality outcomes for vaso-occlusive diseases (e.g., coronary artery disease and stroke) positively correlate with the degree of obesity in patients $(9,10,14,15)$. Collectively, these observations support the notion that obesity increases the risk of thromboembolic disease.

Emergent observations in obese patients have suggested a reciprocal relationship between the thrombin/fibrin(ogen) axis and obesity/metabolic inflammation. A genetic association analysis to evaluate the correlation of thrombophilia mutations with superficial vein thrombosis determined that factor $V^{\text {Leiden }}$ is independently associated with a BMI greater than or equal to $28 \mathrm{~kg} / \mathrm{m}^{2}$ (16). Levels of procoagulant factors (e.g., fibrinogen, factor VII, factor VIII) are higher in obese patients with T2D than in those without T2D (17). Multiple thrombotic risk factors (e.g., APC resistance, protein S/protein C deficiency, prothrombin G20210A) are not only frequently present in patients with 
A

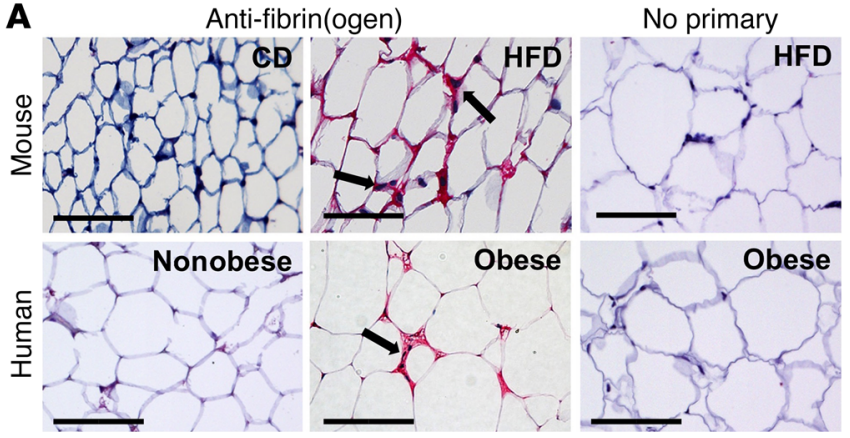

B
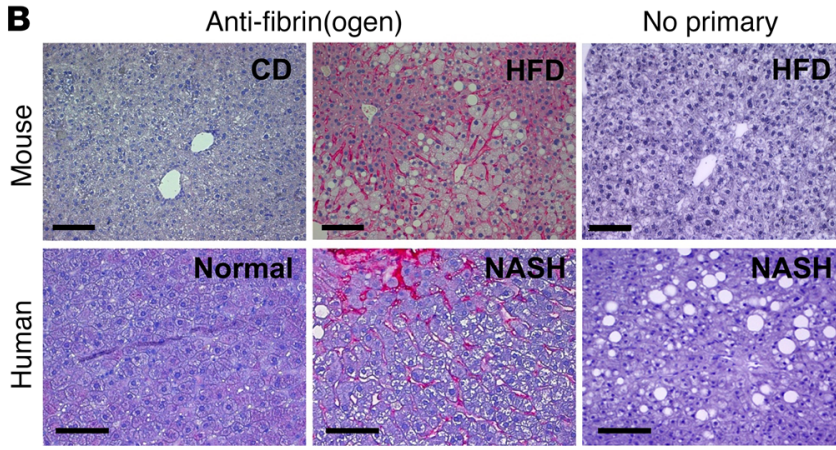

Figure 1. Fibrin(ogen) deposits accumulate in white adipose tissue of HFD-fed mice and obese human patients as well as in liver tissue of HFD-fed mice and patients with NASH. (A) Fibrin(ogen) immunohistochemical staining (red) of white adipose tissue of mice fed either a control diet (CD) or a 60\% high-fat diet (HFD) for 16 weeks as well as visceral adipose tissue of nonobese and obese patients. Note the intense fibrin(ogen) deposits in areas between the hypertrophic adipocytes coincident with macrophages (arrows). (B) Fibrin(ogen) staining (red) of liver tissue from mice fed either a CD or a 60\% HFD for 16 weeks as well as from patients with normal liver histology or obese patients with NASH. Scale bars: $100 \mu \mathrm{m}$.

NAFLD, but are associated with the severe form of this disease, nonalcoholic steatohepatitis (NASH), and advanced liver fibrosis (18). Although these association studies are highly intriguing and have serious potential clinical implications, significant knowledge gaps remain, including whether elevated thrombin activity and adipose fibrin deposits are causative factors in obesity and the identification of potential mechanisms linking thrombin and fibrin to obesity sequelae.

Here, we directly explored the hypothesis that fibrin(ogen) and thrombin activity exacerbate diet-induced obesity, metabolic inflammation, and associated sequelae. To test this hypothesis, mice carrying genetically imposed deficiencies or functional mutations in fibrinogen, factor XIII, thrombomodulin, or protease-activated receptor-1 (PAR1) were analyzed for changes in body weight gain and altered inflammatory and metabolic parameters in both adipose tissue and liver. Our findings provide a mechanistic link between elevated procoagulant function and fibrin(ogen)-driven inflammation in adipose and liver to exacerbation of obesity and obesity-associated sequelae. Further, in proof-of-concept studies using the direct thrombin inhibitor dabigatran etexilate, we demonstrate that inhibiting thrombin activity ameliorates the development of obesity and severity of associated sequelae.

\section{Results}

Fibrinogen drives high-fat diet-induced obesity through the $\gamma^{390-396}$ $\alpha_{M} \beta_{2}$-binding motif. To investigate whether fibrin(ogen) could contribute to diet-induced obesity, fibrin(ogen) immunohistochemistry was performed on epididymal white adipose tissue and liver sections from WT mice fed either a control diet (CD) or a high-fat diet (HFD) for 16 weeks. Little to no fibrin(ogen) deposits were detected in either adipose (Figure 1A) or liver (Figure 1B) from mice fed a CD. However, robust fibrin(ogen) deposits were found throughout the extravascular spaces between hypertrophic adipocytes in HFD-fed mice (Figure 1A). The most intense fibrin(ogen) staining appeared to be in areas between adipocytes in regions with macrophage accumulation in what have been termed "crown" structures (19). Fibrin(ogen) staining was present throughout the liver sinusoids of HFD-fed mice that showed evidence of steatosis (Figure 1B). Importantly, sections of visceral adipose tissue from obese patients revealed an identical pattern with extravascular fibrin(ogen) deposits present in the adipose tissue and most intense in macrophage-rich "crown" structures (Figure 1A). Extravascular fibrin(ogen) deposits were absent in visceral adipose from nonobese individuals. Similarly, liver sections from obese patients with NASH displayed robust fibrin(ogen) deposits in the sinusoids, whereas liver sections with a normal histological presentation were fibrin(ogen)-free (Figure 1B). Collectively, these findings support the concept that fibrin(ogen) could contribute to obesity pathogenesis in both humans and mice.

To gain mechanistic insight into the role of fibrinogen in HFD-induced obesity, we used 2 complementary mouse strains expressing function-selective variants of fibrin(ogen): (a) Fgg 390396A (hereafter termed Fib ${ }^{390-396 A}$ ) mice (20), which express a mutant form of fibrinogen that has normal clotting function but lacks the binding motif for the leukocyte integrin receptor $\alpha_{\mathrm{M}} \beta_{2}$, and (b) Fgg 45 (hereafter termed Fiby $\gamma^{45}$ ) mice (21), carrying a homozygous deletion in fibrinogen for the final 5 amino acids of the $\gamma$ chain that encode the platelet integrin $\alpha_{\mathrm{II}} \beta_{3}$-binding motif. Cohorts of WT and Fib ${ }^{390-396 A}$ mice were fed a CD and HFD (60\% of kcal from fat) for 20 weeks. A marked and statistically significant protection from body weight gain was observed in Fib $\gamma^{390-396 A}$ mice fed the HFD compared with HFD-fed WT mice (Figure 2A), with no effect of genotype on body weight gain in mice fed $\mathrm{CD}$. The difference in body weight between HFD-fed Fib $\gamma^{300-396 A}$ and WT mice was significant as early as 4 weeks after starting on the HFD, and the differential increased throughout the 20-week HFD period (Figure 2, B and $\mathrm{C}$, respectively). Body mass composition analysis revealed that the difference in body weight was secondary to reduced fat mass in Fib $\gamma^{390-396 A}$ mice compared with WT mice, as analyzed at 12 and 16 weeks on HFD (Figure 2, D and E, respectively). Further, the epididymal and inguinal white adipose tissue (eWAT and iWAT, respectively) depots isolated from HFD-fed Fib $\gamma^{390-396 A}$ mice at the end of the challenge period were significantly smaller than eWAT and iWAT from HFD-fed WT mice (Figure 2, F and G).

In contrast, $F i b \gamma^{15}$ mice fed an identical $60 \%$ HFD gained the same amount of weight as WT mice over the course of the challenge period (Figure $2 \mathrm{H})$. Total body weights of CD-fed or HFDfed mice were not different between WT and Fib $\gamma^{15}$ mice at any time point during the challenge period (see Figure 2, I and J, for representative time points of 9 weeks and 16 weeks, respective- 

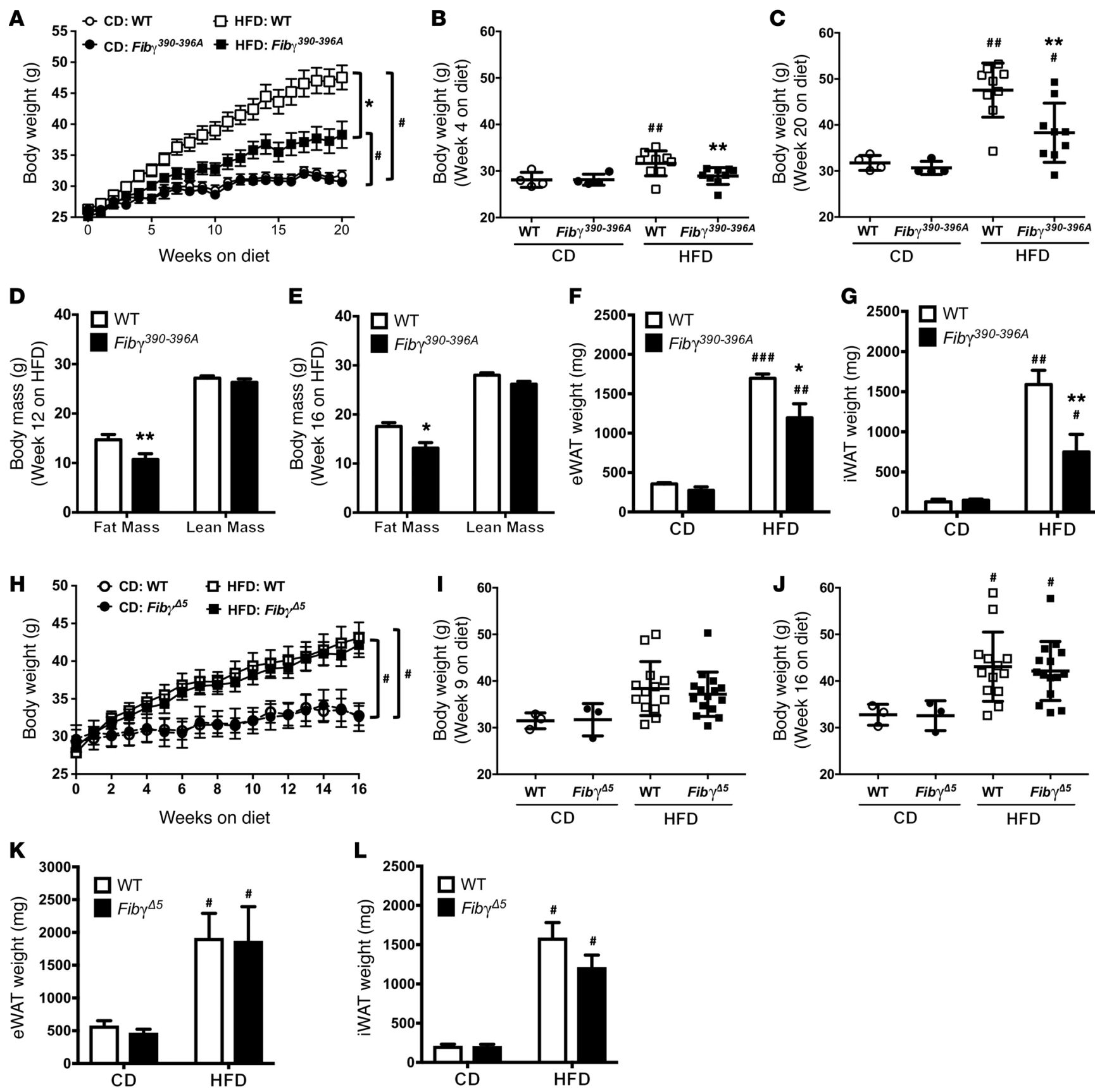

Figure 2. Fib ${ }^{390-3964}$, but not Fib $\gamma^{45}$, mice are protected from the development of HFD-driven obesity. (A-C) WT and Fib $\gamma^{390-3964}$ mice were fed either a CD ( $n=4$ mice per genotype) or a 60\% HFD ( $n=9$ mice per genotype). (A) Mean body weights of WT and fibrinogen $\gamma 390-396 \mathrm{~A}$ (Fib $\gamma^{390-396 A)}$ mice over a 20-week feeding period. (B and C) Distribution of body weights for WT and Fiby ${ }^{390-396 A}$ mice fed the CD or 60\% HFD at week 4 (B) and week 20 (C) on diet. (D and E) Analysis of body mass composition of 60\% HFD-fed WT and Fib $\gamma^{390-396 A}$ mice performed at week 12 (D) and week 16 (E). (F and G) Total fat pad weights of eWAT (F) and IWAT (G) for WT and Fib $\gamma^{390-3964}$ mice at week 20. (H) Mean body weights of WT and fibrinogen $\gamma^{\Delta 5}$ (Fib $\gamma^{\wedge 5}$ ) mice fed a CD ( $n=3$ mice per genotype) or 60\% HFD ( $n=14-15$ mice per genotype) over a 16-week feeding period. (I and J) Analysis of individual body weights for WT and Fib $\gamma^{15}$ mice at week 9 (I) and week 16 (J) on diet. (K and $\mathbf{L}$ ) Total fat pad weights of eWAT (K) and iWAT (L) for WT and Fib ${ }^{15}$ mice at week 16 . Data are expressed as the mean \pm SEM. Data were analyzed by 2-way ANOVA with Student-Newman-Keuls post hoc test. ${ }^{*} P<0.05$, ${ }^{*} P<0.01$ for analyses comparing differences between genotypes on the same diet. $\# P<0.05, \# P<0.01, \# \# P<0.001$ for analyses comparing differences between diets with mice of the same genotype.

ly). No genotype-dependent differences in the weight of eWAT (Figure 2K), iWAT (Figure 2L), or liver (not shown) were observed in tissues harvested at the end of the challenge period for either CD-fed or HFD-fed WT and Fib $\gamma^{\wedge 5}$ mice. Collectively, these findings indicate that the $\gamma^{330-396}$ motif of fibrin(ogen) specifically promotes HFD-induced obesity.
To determine whether the protection from HFD-induced weight gain observed in Fib ${ }^{390-396 A}$ mice was limited to the specific diet tested, we evaluated weight gain in mice using a separate set of diets. Here, a HFD formulated to $45 \%$ of kcal from fat was used with a corresponding CD with $10 \%$ of kcal from fat. Similar to analyses using the $60 \%$ HFD, Fib $\gamma^{390-396 A}$ mice fed the 

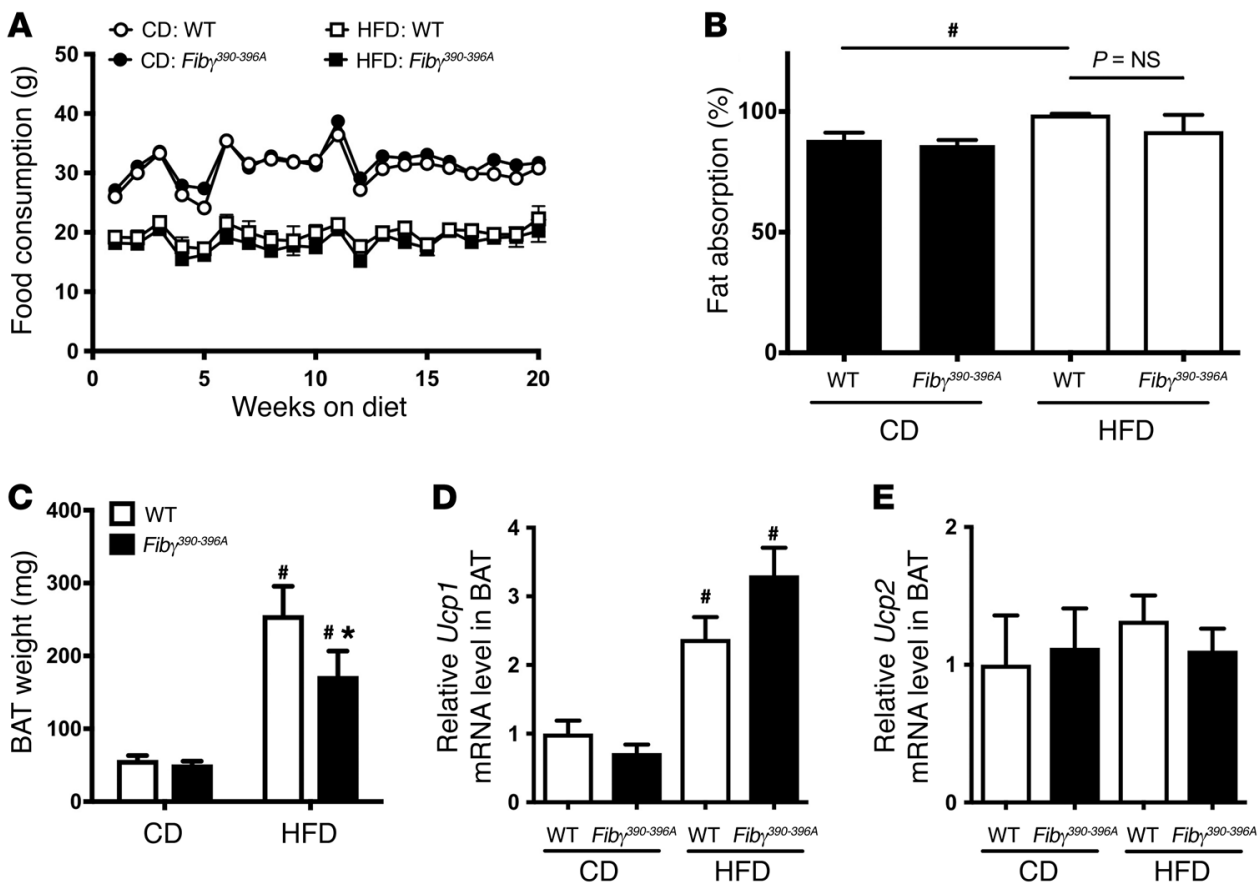

Figure 3. Fib $\gamma^{390-3964}$ mice consume the same amount of food, have similar intestinal fat absorption, and have reduced brown adipose tissue weight following HFD challenge compared with WT mice. (A) Food consumption based on weight of food consumed per mouse per week for WT and Fib $\gamma^{390-396 A}$ mice fed a CD ( $n=4$ per genotype) and a 60\% HFD ( $n=9$ per genotype). (B) Intestinal fat absorption using the behenic acid assay (see Methods) for WT and Fib $\gamma^{390-396 A}$ mice fed either a CD or a 60\% HFD for 16 weeks ( $n=4$ mice per genotype per diet). (C) Brown adipose tissue (BAT) weights of WT and Fib $\gamma^{390-396 A}$ mice fed either a CD ( $n=4$ mice per genotype) or a $60 \%$ HFD ( $n=9$ mice per genotype). (D and E) Quantitative reverse transcriptase PCR (RT-PCR) analysis of BAT from WT and Fib $\gamma^{390-396 A}$ mice fed a CD or a HFD for Ucp1 (D) or Ucp2 (E). Data are expressed as the mean \pm SEM. Data were analyzed by 2-way ANOVA with Student-Newman-Keuls post hoc test. ${ }^{*} P<0.05$ for analyses comparing differences between genotypes on the same diet. ${ }^{\#} P<0.05$ for analyses comparing differences between diets with mice of the same genotype.

$45 \%$ HFD gained more weight than those on CD, but, importantly, gained significantly less weight compared with WT mice fed the $45 \%$ HFD (Supplemental Figure 1A; supplemental material available online with this article; https://doi.org/10.1172/ JCI92744DS1). The significant difference in weight gain was first apparent at 7 weeks on HFD, and the differential increased to the end of the challenge period at 20 weeks (Supplemental Figure 1, B and C, respectively). Analysis of eWAT and iWAT revealed that these tissues had a significantly greater mass in WT mice fed the 45\% HFD compared with Fib $\gamma^{390-396 A}$ mice (Supplemental Figure 1, D and E, respectively).

The difference in weight gain displayed by the $F i b \gamma^{390-396 A}$ mice on either the $60 \%$ or the $45 \%$ HFD could not be explained by differences in food consumption (Figure $3 \mathrm{~A}$ and data not shown, respectively). In addition, the weight gain difference did not appear to be linked to differences in intestinal fat absorption, as no genotype-dependent difference in fat absorption was detected in mice fed a CD or HFD for 16 weeks (Figure 3B). However, HFD-fed WT mice overall displayed significantly higher fat absorption than CD-fed mice (Figure 3B), as previously described (22). Similarly, the difference in HFD-induced weight gain did not appear to be linked to differential compensatory changes in brown adipose tissue (BAT). As expected, the BAT weight in WT mice was significantly higher in HFD-fed animals than in CD-fed animals (Figure 3C). A significant increase was also observed for Fib $\gamma^{390-396 A}$ mice when comparing CD- and HFD-fed animals.
However, similarly to WAT, the BAT weights of Fib $\gamma^{390-396 A}$ mice fed a HFD were significantly lower than those in HFD-fed WT mice (Figure 3C). Additionally, no genotype-dependent differences in gene expression of key mediators of thermogenesis (i.e., Ucp1 and Ucp2) were observed in HFD-fed WT and Fib $\gamma^{390-396 A}$ mice (Figure 3, D and E, respectively).

The fibrinogen $\gamma^{390-396}$ motif supports binding to the coagulation transglutaminase factor XIII in addition to binding the leukocyte integrin receptor $\alpha_{M} \beta_{2}$ (23). To determine whether the decrease in body weight following HFD feeding observed in Fib $\gamma^{390-396 A}$ mice relative to WT mice involved factor XIII, mice deficient in the catalytic A subunit of factor XIII ( $\left.\mathrm{F} \mathrm{Fa1}^{-/-}\right)$and WT mice were fed the $60 \%$ HFD and CD for 20 weeks. In contrast to results with $\mathrm{Fib \gamma}{ }^{390-396 \mathrm{~A}}$ mice, weight gain of $\mathrm{F} 13 \mathrm{a1^{-/ }}$ mice was virtually identical to that of WT mice (Supplemental Figure 2, A-C). Similarly, there were no genotype-dependent differences in the weight of eWAT or liver for either CD-fed or HFD-fed WT and $\mathrm{F}_{13 \mathrm{al}} \mathrm{I}^{-/}$mice (Supplemental Figure 2, D and E, respectively).

Fib $\gamma^{390-396 A}$ mice are protected from the development of HFDinduced systemic and local inflammation. To determine whether the lower body weight gain and fatty liver disease observed in HFD-fed Fib $\gamma^{390-396 A}$ mice were indeed linked to a reduction in systemic and local inflammation, we first measured circulating proinflammatory cytokines/chemokines in plasma. TNF- $\alpha$, MCP-1, and IL-17A have each been linked to exacerbation of obesity-associated sequelae (24-28), and each was significantly 
A
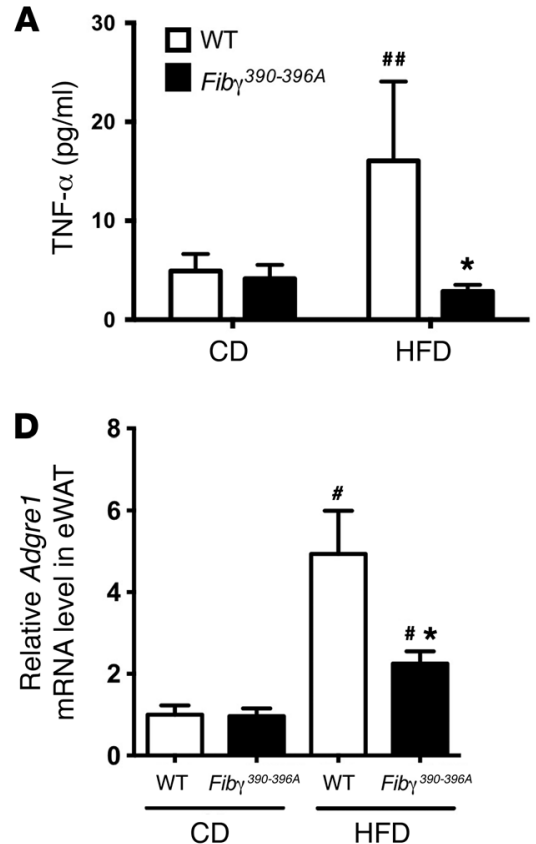

B

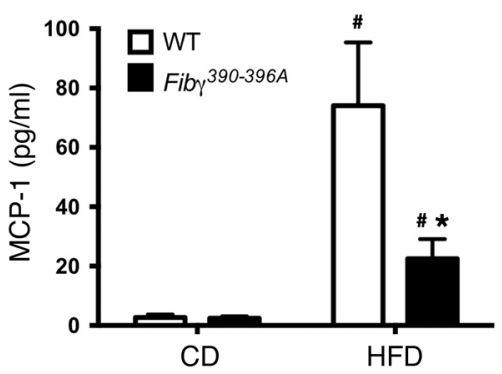

E

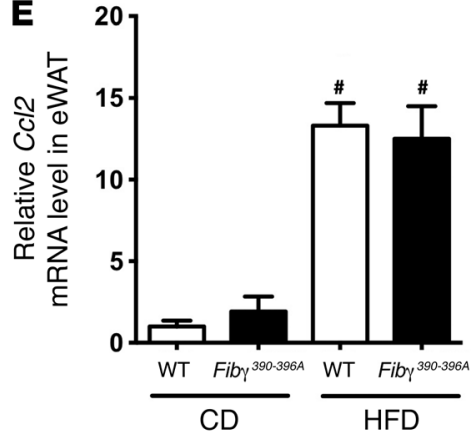

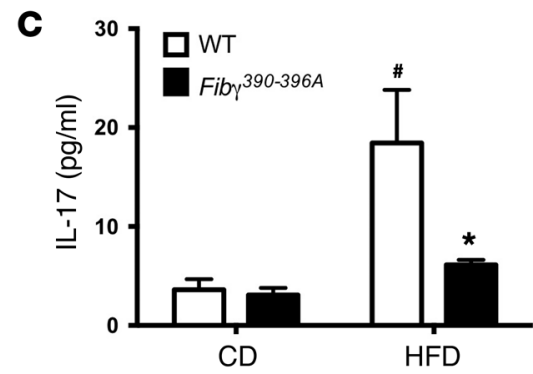

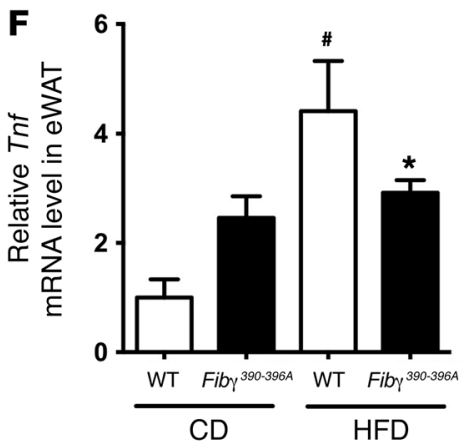

Figure 4. Fib $\gamma^{390-396 A}$ mice develop significantly diminished HFD-induced systemic and adipose inflammation. WT and Fib $\gamma^{390-396 A}$ mice were fed a CD ( $n=4$ mice per genotype) or a 60\% HFD ( $n=11-12$ mice per genotype) for 20 weeks. (A-C) Circulating levels of TNF- $\alpha$ (A), MCP-1 (B), and IL-17 (C) in plasma. (D-F) Levels of mRNA in eWAT for the macrophage marker F4/80 (Adgre1) (D), the macrophage chemokine MCP-1 (Cc/2) (E), and the proinflammatory cytokine TNF- $\alpha(\operatorname{Tnf})(\mathbf{F})$ were determined by quantitative RT-PCR. Data are expressed as the mean $\pm \mathrm{SEM}$. Data were analyzed by 2-way ANOVA with Student-Newman-Keuls post hoc test. ${ }^{*} P<0.05$ for analyses comparing differences between genotypes on the same diet. ${ }^{\#} P<0.05$, \#\# $P<0.01$ for analyses comparing differences between diets with mice of the same genotype.

elevated in HFD-fed WT mice compared with CD-fed WT mice (Figure 4, A-C, respectively). However, circulating levels of these proinflammatory mediators were significantly lower in HFD-fed Fib $\gamma^{390-396 A}$ mice compared with WT mice (Figure 4, A-C). Proinflammatory macrophages and effector molecules have been linked to exacerbation of adiposity in diet-driven obesity (29-31). Thus, we examined expression of genes encoding inflammatory markers in eWAT. Here, eWAT mRNA levels of F4/80 (Adgre1) and TNF- $\alpha$ (Tnf) were significantly elevated in HFD-fed WT mice compared with CD-fed WT mice but significantly lower in HFD-fed Fib $\gamma^{390-396 A}$ mice (Figure 4, D and F). MCP-1 mRNA levels (Ccl2) in eWAT were not different between HFD-fed WT and Fib $\gamma^{390-396 A}$ mice, although each was elevated in comparison with corresponding CD-fed animals (Figure 4E).

To further evaluate the effect of the fibrinogen $\gamma^{390-396 \mathrm{~A}}$ mutation on adipose inflammation, eWAT tissue sections from CDand HFD-fed mice were stained for adipose tissue macrophages (ATMs) using the macrophage marker F4/80. In CD-fed animals, $\mathrm{F} 4 / 80^{+}$cells were sparse in both genotypes (Figure $5 \mathrm{~A}$ ). In contrast, $\mathrm{F} 4 / 80^{+}$cells were readily detected in eWAT from HFD-fed WT mice and appeared clustered in "crown" structures (Figure 5A). F4 $/ 80^{+}$cells were also observed in HFD-fed Fib $\gamma^{390-396 \mathrm{~A}}$ mice, but appeared fewer in number. Quantification of $\mathrm{F} 4 / 80^{+}$cells indicated a significant increase in cell number for HFD-fed mice over $\mathrm{CD}$-fed mice for both genotypes, although the number of $\mathrm{F} 4 / 80^{+}$ cells was significantly reduced in HFD-fed Fib $\gamma^{390-396 A}$ mice compared with HFD-fed WT mice (Figure 5B). Consistent with the notion that fibrin(ogen)-driven macrophage function was promoting adiposity, quantification of adipocyte size revealed a strikingly similar pattern, with adipocyte area being small in CD-fed mice, significantly elevated in HFD-fed WT mice, but comparatively significantly lower in HFD-fed Fib $\gamma^{390-396 A}$ mice (Figure 5C). An analysis was performed for neutrophils in eWAT, but few if any positive cells were identified in any of the eWAT tissue samples (data not shown). Collectively, these results suggest that Fib $\gamma^{390-396 A}$ mice are protected from the development of inflammation associated with obesity as well as obesity itself.

Fib $\gamma^{390-396 A}$ mice are protected from the development of HFDinduced fatty liver disease and glucose dysmetabolism. To determine whether reduced weight gain in HFD-fed Fib $\gamma^{390-396 A}$ mice was linked to protection from the development of obesity sequelae, we first evaluated fatty liver disease. Histological analysis of liver sections revealed significant steatosis in HFD-fed WT mice but not in HFD-fed Fib $\gamma^{390-396 A}$ mice (Figure 6A). This tracked with liver weights of HFD-fed Fib $\gamma^{390-396 A}$ mice that were significantly lower compared with those of HFD-fed WT mice (Figure 6B). Fibrin(ogen) deposits were absent in livers from CD-fed mice but were readily detected in the liver sinusoids of HFD-fed WT mice around hepatocytes displaying histological evidence of steatosis (Figure 6A). Fibrin(ogen) deposits were detected in the liver sinusoids of HFD-fed Fib $\gamma^{390-396 A}$ mice (Figure 6A), but like the diminished steatosis, these fibrin(ogen) deposits were also significantly reduced (Figure 6C). Matching the changes in liver histology, liver triglyceride levels were significantly elevated in HFD-fed WT mice compared with Fib $\gamma^{390-396 A}$ mice (Figure 6D). Notably, the fatty liver disease in HFD-fed WT mice was associated with significant hepatocellular damage as indicated by elevated serum alanine aminotransferase (ALT; Figure 6E). HFD-fed Fib $\gamma^{390-396 A}$ mice 
A

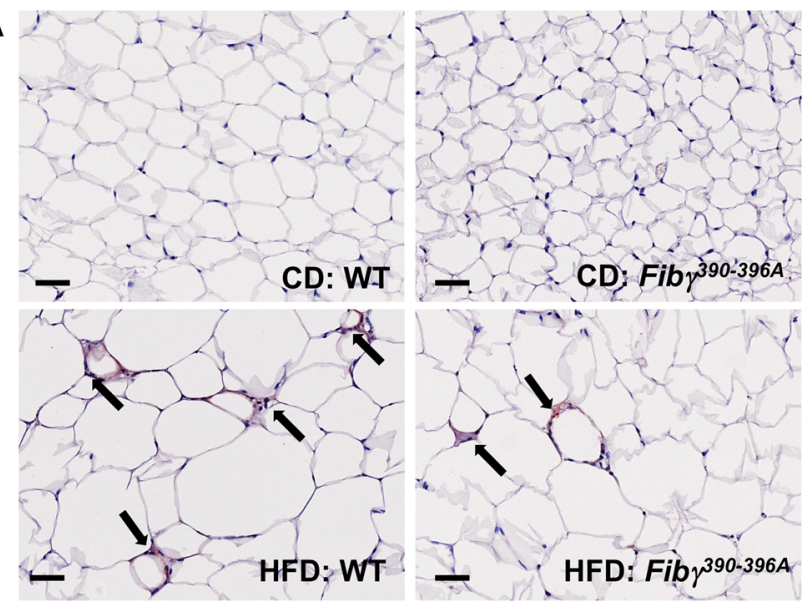

B

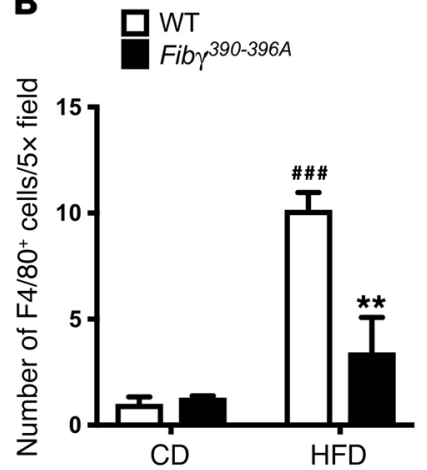

C

口WT

Fib $\gamma^{390-396 A}$

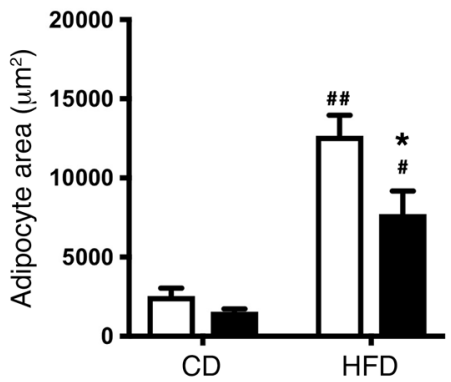

Figure 5. Fib $\gamma^{390-396 A}$ mice fed a HFD for 20 weeks have significantly fewer F4/80+ macrophages and reduced adipocyte size relative to HFD-fed WT mice. (A) Tissue sections of eWAT from WT and Fib $\gamma^{390-396 A}$ mice fed either a CD or a 60\% HFD for 20 weeks were stained by immunohistochemistry for the macrophage marker $\mathrm{F} 4 / 80$. Note that $\mathrm{F} 4 / 80^{+}$cells (arrows) accumulated in "crown-like" structures similar to the fibrin(ogen) deposits shown in Figure 1 . Scale bars: $50 \mu \mathrm{m}$. (B) Quantification of the number of F4/80+ cells per $\times 5$ high-powered field in eWAT of WT and Fib $\gamma^{390-396 A}$ mice fed CD ( $n=3$ per genotype) and HFD ( $n=5$ per genotype). Note that 10 fields were evaluated per sample. (C) Quantification of adipocyte area in the eWAT of WT and Fib $\gamma^{390-3964}$ mice fed CD ( $n=3$ per genotype) and HFD ( $n=5$ per genotype). Note that the area of 50 adipocytes per sample was determined. Data in $\mathbf{B}$ and $\mathbf{C}$ are presented as mean \pm SEM. Data were analyzed by 2-way ANOVA with Student-Newman-Keuls post hoc test. ${ }^{*} P<0.05$, ${ }^{* *} P<0.01$ for analyses comparing differences between genotypes on the same diet. ${ }^{\#} P<0.05,{ }^{\# \#} P<0.01,{ }^{\# \#} P<0.001$ for analyses comparing differences between diets with mice of the same genotype.

were protected from hepatocellular injury as indicated by serum ALT activity equivalent to that of CD-fed mice (Figure 6E). Hepatic inflammatory markers were also examined. Levels of Adgre1 mRNA were elevated in HFD-fed mice compared with CD-fed animals, but no genotype-dependent difference was observed (Figure 6F). Significantly reduced hepatic mRNA levels of $\mathrm{Ccl} 2$ and $T n f$ were observed in HFD-fed Fib $\gamma^{390-396 A}$ mice compared WT mice (Figure 6, G and $\mathrm{H}$, respectively). The potential molecular basis of significantly diminished steatosis in Fib $\gamma^{390-396 A}$ mice was revealed through analysis of gene products associated with lipid accumulation/lipogenesis. Hepatic mRNA levels of the nuclear receptor PPAR $\gamma$ (Pparg), the fatty acid sensor CIDEA (Cidea), and the fatty acid transporter CD36 (Cd36) were each significantly lower in HFD-fed Fib $\gamma^{390-396 A}$ mice compared with WT mice (Figure 6, I-K, respectively).

HFD-mediated changes in glucose metabolism were also determined. Analysis of fasting blood glucose levels showed that HFD-fed mice of both genotypes had significantly higher blood glucose levels compared with CD-fed mice (Figure 7A). Importantly, glucose clearance was impaired by HFD challenge, but clearance was significantly more efficient in HFD-fed Fibr ${ }^{390-396 A}$ mice compared with HFD-fed WT mice as analyzed by 2-hour glucose tolerance tests (Figure 7B). Similarly, HFD-fed WT mice displayed a profound loss of insulin sensitivity as shown by insulin tolerance tests compared with CD-fed mice. Insulin sensitivity in HFD-challenged Fib $\gamma^{390-396 A}$ mice was significantly improved compared with that in HFD-fed WT mice (Figure 7C). Accordingly, we observed significantly elevated fasting insulin levels in HFD-fed mice compared with $\mathrm{CD}$ mice, but insulin levels were significantly lower in HFD-fed Fib $\gamma^{390-396 A}$ mice compared with HFD-fed WT mice (Figure 8A). Consistent with differential body weights, the adipokines leptin and resistin were significantly higher in HFDfed WT mice compared with HFD-fed Fib $\gamma^{390-396 A}$ mice at 20 weeks
(Figure 8, B and C, respectively). Interestingly, leptin, resistin, and adiponectin were each significantly lower in Fib $\gamma^{390-396 A}$ mice compared with WT mice fed a CD (Figure 8, B-D, respectively).

Mice with a genetically imposed procoagulant state exhibit exacerbated obesity following a HFD challenge. The effect of diet and fibrinogen genotype on coagulation parameters was assessed by measurement of TAT, D-dimer, and platelet factor 4 (PF4) in the plasma of CD-fed and HFD-fed WT and Fib ${ }^{390-396 A}$ mice. HFD challenge resulted in significantly higher TAT and D-dimer levels in WT mice compared with CD-fed mice (Table 1). Notably, plasma levels of both of these coagulation markers were also elevated in Fib $\gamma^{390-396 A}$ mice fed a HFD compared with a $\mathrm{CD}$ (Table 1). Plasma levels of the platelet activation marker PF4 were similar across diet and genotype (Table 1), suggesting that differential platelet activation was not the basis of fibrinogen genotype-dependent differences in HFD-induced obesity.

To investigate whether enhanced procoagulant function itself could alter the development of diet-induced obesity and obesity-associated disease sequelae, we evaluated HFDinduced obesity in mice homozygous for a Glu404Pro mutation in the thrombomodulin receptor $\left(T h b d^{\text {Pro }}\right)$. $T h b d^{\text {Pro }}$ mice exhibit a hypercoagulable state due to significantly reduced thrombin binding and disrupted thrombomodulin-dependent protein $\mathrm{C}$ activation by the mutant receptor. Both Thbd ${ }^{\text {Pro }}$ and WT animals gained significantly more weight on the HFD than cohorts of mice fed a CD (Supplemental Figure 3A). However, Thbd ${ }^{\text {Pro }}$ mice fed a HFD gained significantly more weight than WT mice fed a HFD (Supplemental Figure 3A). The significant genotype-dependent difference in body weight was first apparent at approximately 11 weeks on the HFD (Supplemental Figure 3B) and persisted through 16 weeks (Supplemental Figure 3C). MRIbased body mass composition revealed a significant increase in fat mass, but not lean mass, in HFD-fed $T h b d^{\text {Pro }}$ mice at 12 and 16 weeks (Supplemental Figure 3, D and E, respectively). The 
A
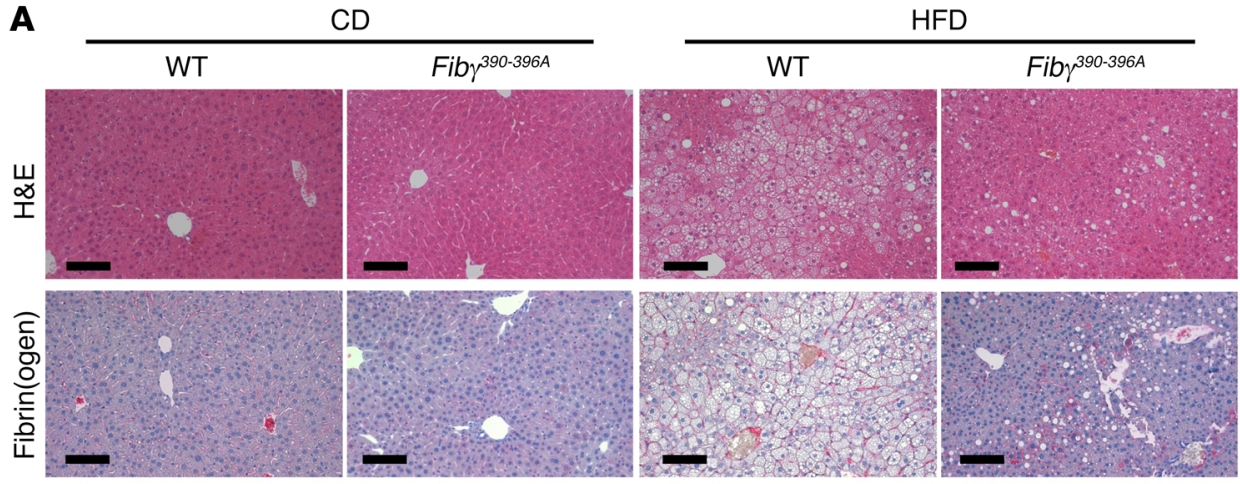

B

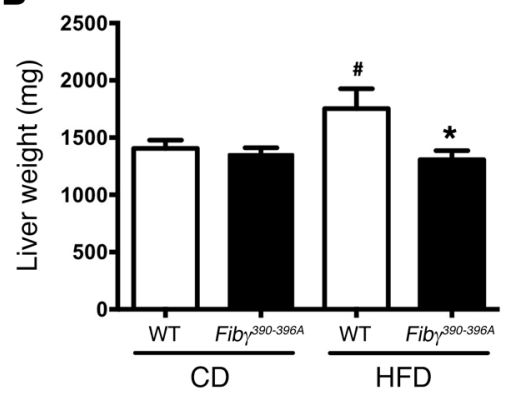

D

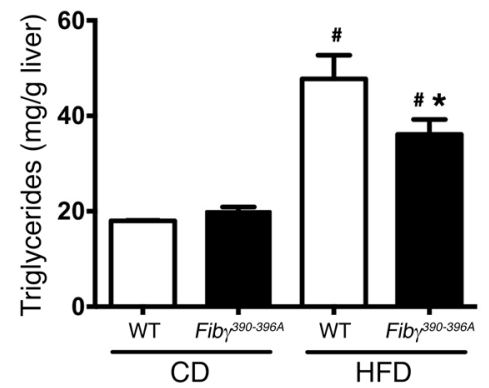

C

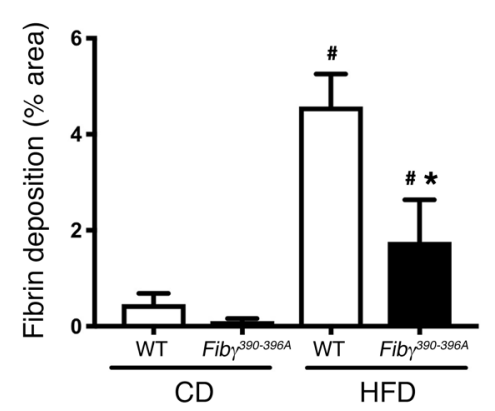

E

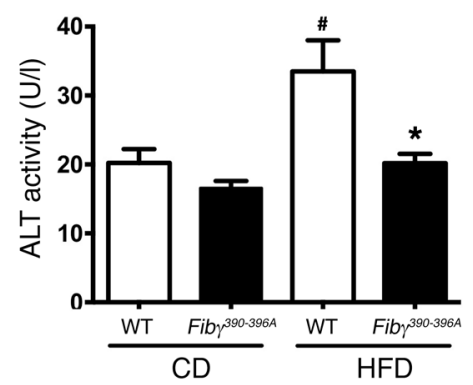

Figure 6. Fiby ${ }^{390-396 A}$ mice are protected from the development of HFD-driven fatty liver disease and hepatocellular injury. WT and Fiby ${ }^{390-396 A}$ mice were fed either a CD ( $n=4$ per genotype) or a 60\% HFD ( $n=12$ per genotype) for 20 weeks. (A) Representative H\&E-stained and fibrin(ogen) (red) immunohistochemistry-stained sections of liver tissue. Note that liver tissue from HFD-fed WT mice revealed evidence of hepatic steatosis and sinusoidal fibrin(ogen) deposits. Scale bars: $100 \mu \mathrm{m}$. (B) Total liver weights of mice following the 20-week diet challenge. (C) Quantification of fibrin deposits in CD-fed ( $n=4$ per genotype) and HFD-fed ( $n=8$ per genotype) WT and Fib $\gamma^{390-396 A}$ mice. Data are presented as the mean \pm SEM of the percent area of staining per high-powered field. (D) Analysis of liver triglyceride content confirmed significantly diminished hepatic steatosis in HFD-fed Fib ${ }^{390-396 A}$ mice relative to WT animals. (E) Analysis of circulating alanine aminotransferase (ALT) in CD- and HFD-fed WT and Fib $\gamma^{390-396 A}$ mice indicated significantly reduced hepatocellular damage in HFD-fed Fib $\gamma^{390-396 A}$ mice. (F-K) Hepatic levels of mRNA in liver tissue encoding the genes Adgre1 (F), Ccl2 (G), Tnf (H), Pparg (I), Cidea (J), and Cd36 (K) were determined by quantitative RT-PCR. Data are expressed as the mean \pm SEM. Data were analyzed by 2-way ANOVA with Student-Newman-Keuls post hoc test. ${ }^{*} P<0.05$, ${ }^{* *} P<0.01,{ }^{* * *} P<0.001$ for analyses comparing differences between genotypes on the same diet. ${ }^{\#} P<0.05$, ${ }^{\#} P<0.01$ for analyses comparing differences between diets with mice of the same genotype.
F

F

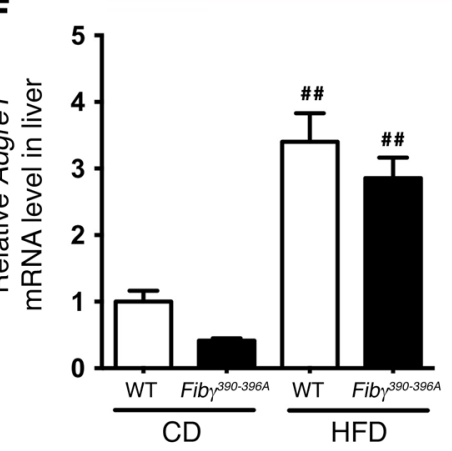

I

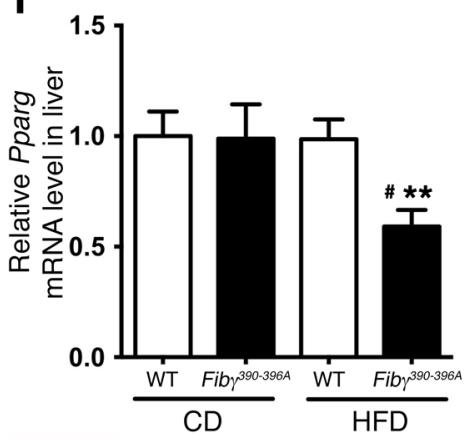

G

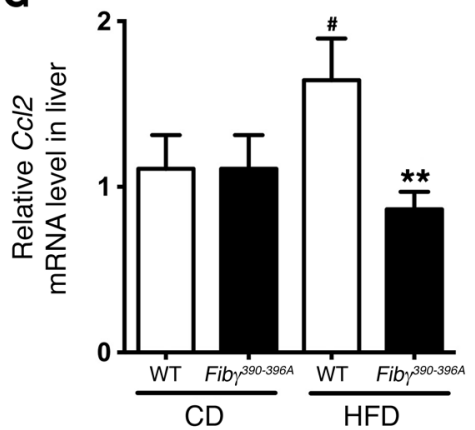

$\mathbf{J}$

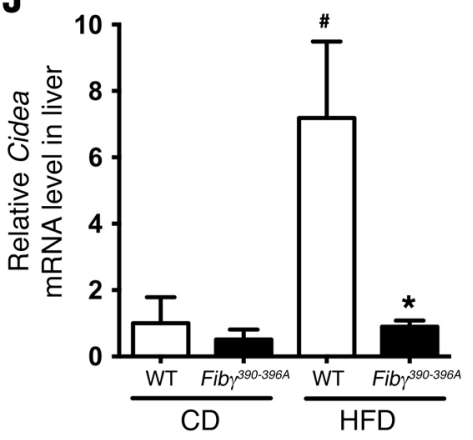

H

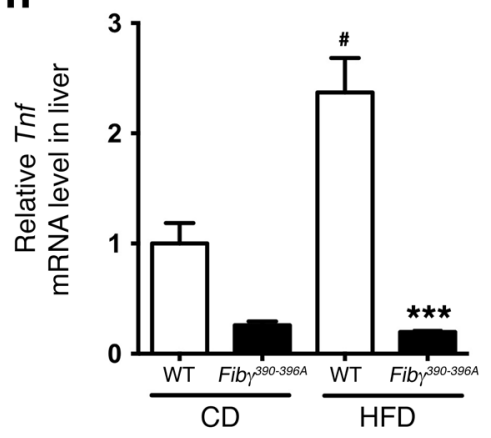

K

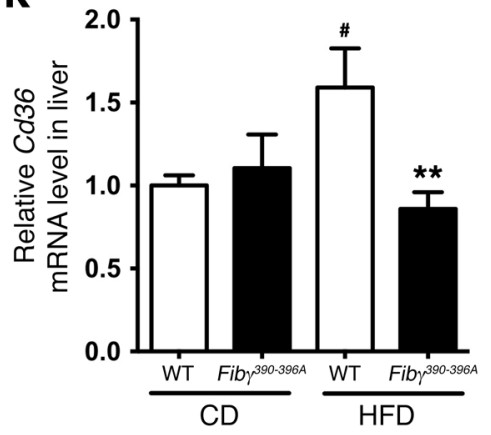


A

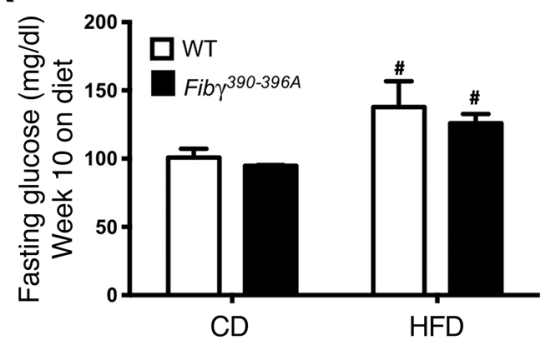

B
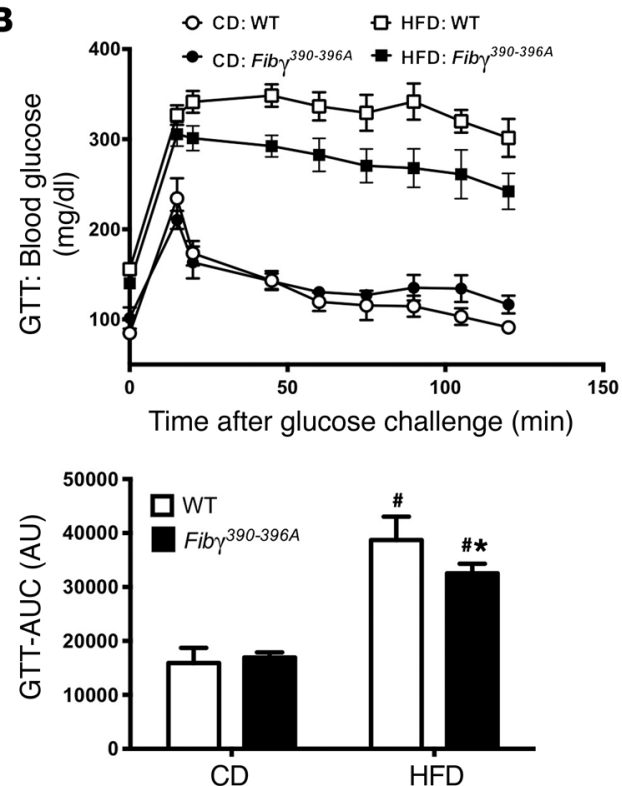

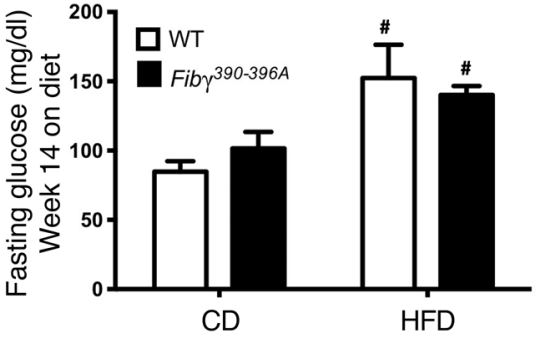

C
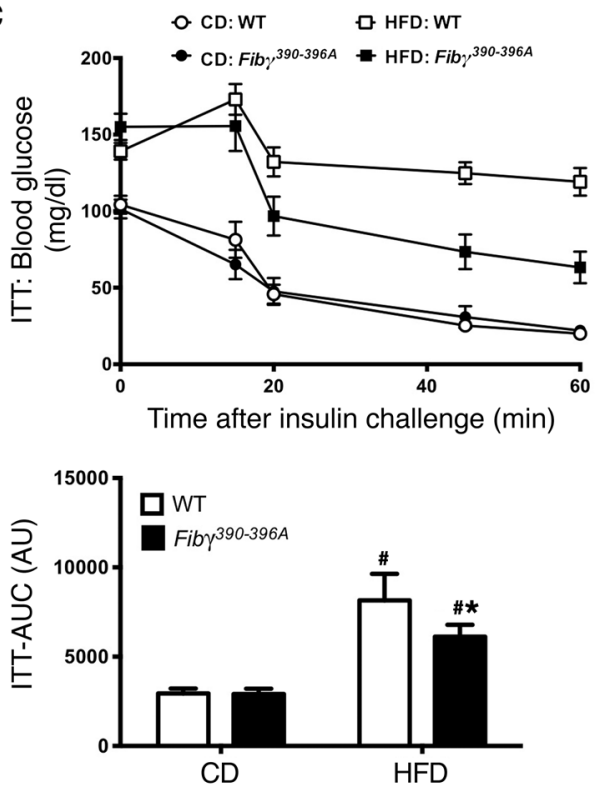

Figure 7. Fib $\gamma^{390-396 A}$ mice are protected from HFD-induced glucose dysmetabolism and insulin resistance. WT and Fib $\gamma^{390-396 A}$ mice were fed a CD $(n=5$ mice per genotype) or a 60\% HFD ( $n=11-12$ mice per genotype) for up to 20 weeks. (A) Fasting blood glucose was taken at week 10 and week 14 on diet. (B and C) A glucose tolerance test (GTT) was performed at week 16 on diet (B), and an insulin tolerance test (ITT) was performed at week 18 on diet (C). The AUC was determined for each individual animal for GTT and ITT. Data are presented as the mean \pm SEM. Data were analyzed by 2-way ANOVA with Student-Newman-Keuls post hoc test. ${ }^{*} P<0.05$ for analyses comparing differences between genotypes on the same diet. ${ }^{\#}<0.05$ for analyses comparing differences between diets with mice of the same genotype.

eWAT weights of HFD-fed Thbd ${ }^{\text {Pro }}$ mice were significantly elevated compared with those of HFD-fed WT mice (Supplemental Figure 3F), and the iWAT weights trended higher (Supplemental Figure $3 G)$. The increase in adiposity for $T_{h b d^{\text {Pro }}}$ mice was associated with increased adipose inflammation. Levels of mRNA for F4/80 (Adgre1), MCP-1 (Ccl2), and TNF- $\alpha$ (Tnf) were each significantly elevated in eWAT of HFD-fed Thbdro mice compared with HFD-fed WT mice (Supplemental Figure 3, H-J).

The liver weight of HFD-fed Thbd ${ }^{\text {Pro }}$ mice was significantly elevated compared with that of HFD-fed WT mice (Supplemental Figure 4A). Microscopic analysis of livers from CD-fed WT and Thbdro mice indicated a normal liver histology (Supplemental Figure 4B), whereas livers from HFD-fed WT mice revealed robust steatosis that was exacerbated in HFD-fed Thbd ${ }^{\text {Pro }}$ mice (Supplemental Figure $4 \mathrm{~B})$. Notably, immunohistochemical analysis of liver sections revealed significant fibrin(ogen) deposits lining the hepatic sinusoids of HFD-fed WT mice, which appeared elevated in liver sections of HFD-fed $T h b d^{\text {Pro }}$ mice in terms of both the area and intensity of staining (Supplemental Figure 4B). Liver sections from CD-fed mice revealed little fibrin(ogen) staining regardless of genotype
(Supplemental Figure 4B). HFD-fed Thbd ${ }^{\text {Pro }}$ mice had significantly higher liver triglyceride levels than WT mice fed a HFD (Supplemental Figure 4C). Similarly, HFD-fed Thbd ${ }^{\text {Pro }}$ mice had significantly higher ALT levels relative to HFD-fed WT mice and mice fed a CD (Supplemental Figure 4D). Inflammatory genes in the liver were not significantly elevated in HFD-fed Thbd $d^{\text {Pro }}$ mice compared with HFD-fed WT mice (Supplemental Figure 4, E-G). However, hepatic mRNA levels of the lipogenic genes Pparg, Cidea, and Cd36 (Supplemental Figure 4, H-J) were significantly higher in HFD-fed $T h b d^{\text {Pro }}$ mice compared with HFD-fed WT mice. Taken together, these data indicate that defective thrombomodulin function exacerbates obesity-induced fatty liver disease.

HFD studies in $T h b d^{\text {Pro }}$ and Fib $\gamma^{390-396 A}$ mice suggested that (a) dysregulated thrombin activity promotes obesity and (b) the mechanism of thrombin-driven obesity is linked to fibrinogen. To determine whether the mechanisms of thrombin-driven HFD-induced obesity could also be mediated through the thrombin substrate protease-activated receptor-1 (PAR1), we analyzed HFD-induced obesity using PAR1-deficient mice $\left(F 2 r^{--}\right)$. Cohorts of $F 2 r^{-/-}$mice and WT mice were fed the same 
A
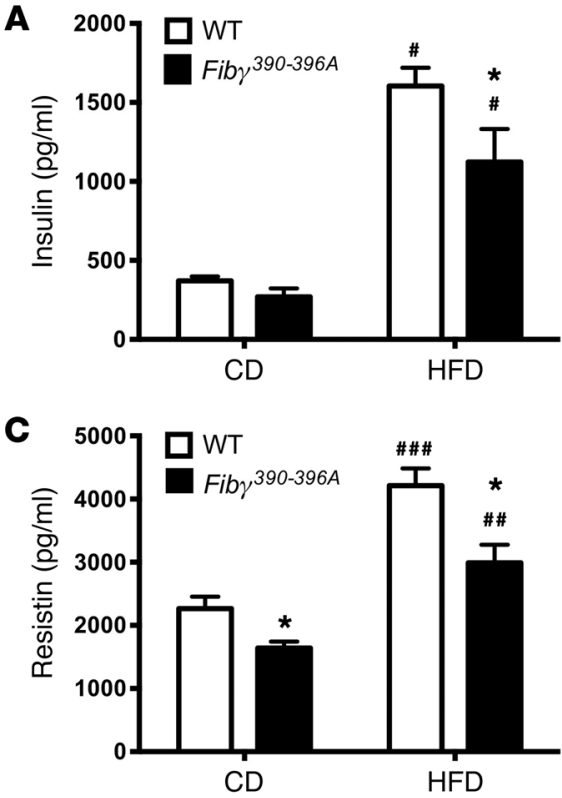

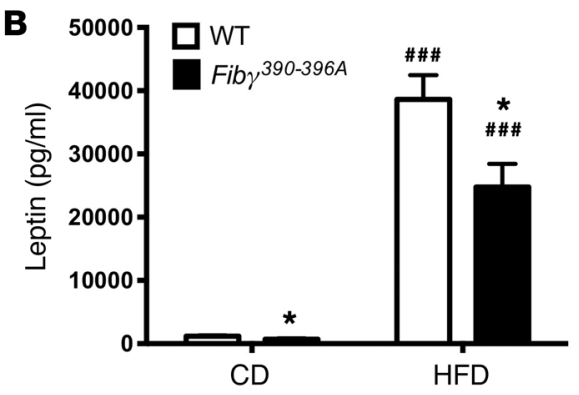

D

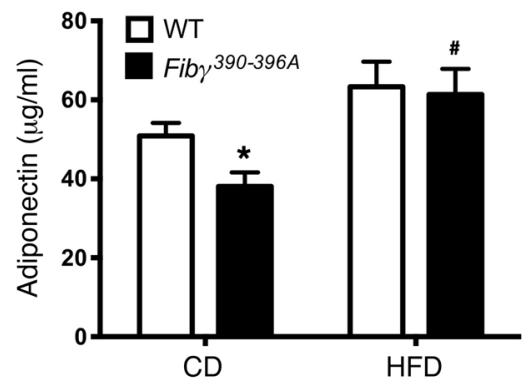

Figure 8. Fib $\gamma^{390-396 A}$ mice have significantly diminished HFD-induced levels of circulating adipolkines. WT and Fib $\gamma^{390-396 A}$ mice were fed a CD ( $n=5$ mice per genotype) or a 60\% HFD ( $n=11-12$ mice per genotype) for 20 weeks. Mice were fasted 6 hours before collection of plasma and determination of insulin (A), leptin (B), resistin (C), and adiponectin (D) levels. Data are expressed as the mean \pm SEM. Data were analyzed by 2 -way ANOVA with StudentNewman-Keuls post hoc test. ${ }^{*} P<0.05$ for analyses comparing differences between genotypes on the same diet. ${ }^{\#}<0.05$, \#\# $P<0.01$, \#\#\# $P 0.001$ for analyses comparing differences between diets with mice of the same genotype.

HFD and CD used in Fib $\gamma^{390-396 A}$ and Thbd $^{\text {Pro }}$ studies (i.e., 60\% of kcal from fat). Consistent with previous studies (32), no genotype-dependent difference in weight gain was observed in WT and $\mathrm{F}^{-r^{-/}}$mice on either diet (Supplemental Figure 5A). Indeed, the weights of individual HFD-fed WT and $F 2 r^{-/}$mice were comparable at multiple weeks on the diet (Supplemental Figure 5, B and C). Further, the weights of eWAT and livers isolated from WT and $\mathrm{F}_{2} \mathrm{r}^{-/}$mice at the end of the challenge period were not different between CD-fed or HFD-fed animals (Supplemental Figure 5, D and E, respectively).

The direct thrombin inhibitor dabigatran etexilate protects mice from HFD-induced obesity development and successfully treats established obesity sequelae. In agreement with an earlier report (33), we found that prophylactic administration of dabigatran etexilate (DE) (7.5 mg/g diet) over the course of 20 weeks attenuated body weight gain (and fat mass) in mice fed a HFD (60\% of kcal from fat) (Figure $9, \mathrm{~A}-\mathrm{C}$ ), indicating protection from obesity in 2 distinct HFD-induced obesity models. Our working hypothesis was that the beneficial effect of DE was through prevention of thrombin-mediated fibrin deposition in WAT of HFD-fed mice. DE treatment significantly reduced levels of high-molecular weight fibrin species (defined as $>350 \mathrm{kDa}$ ) in extracts of eWAT from HFD-fed mice, as determined by capillary-based Western blotting (Figure 9D). The absence of these fibrin species in plasma clots formed using $\mathrm{F} 13 \mathrm{a1^{-/ }}$ plasma confirmed the identity of these species as prototypical cross-linked fibrin.

To address whether DE could rescue established obesity, mice were fed CD or HFD without the drug for 12 weeks to induce obesity (Table 2). The mice then continued to receive the same diet for an additional 8 weeks, but were randomized to receive diet with or without DE during this rescue arm of the study (Figure 9E). As expected, HFD-fed mice without DE continued to gain more weight and fat mass compared with mice fed CD (Figure $9 \mathrm{~F})$. Importantly, the extent of weight gain in weeks $12-20$ was markedly reduced in HFD-fed mice receiving DE compared with mice receiving HFD without DE, a change attributable to reduced accumulation of fat mass, not lean mass (Table 3). Differences in body weight could not be accounted for by differences in food consumption (not shown). Serum ALT activity increased dramatically in mice fed HFD from weeks 12 to 20, denoting the onset of hepatocellular injury and more severe NAFLD (Table 3). Serum ALT failed to increase in HFD-fed DE-treated mice (Table 3), indicating that in addition to limiting HFD-induced adiposity, DE treatment in established obesity suppresses the progression of existing NAFLD. Histological assessment of liver tissue from mice at the end of the 20-week evaluation period indicated lower extent of microvesicular steatosis, the form of lipid accumulation spe-

Table 1. Analysis of TAT, D-dimer, and PF4 in plasma from WT and Fib $\gamma^{390-396 A}$ fed a CD or HFD for 20 weeks

CD

HFD

\begin{tabular}{lcccc} 
Plasma marker & WT & Fib $\boldsymbol{y}^{390-396 A}$ & WT & Fib $\boldsymbol{y}^{390-396 A}$ \\
\hline TAT $(\mu \mathrm{g} / \mathrm{l})$ & $9.1 \pm 1.1$ & $9.7 \pm 1.6$ & $17.6 \pm 3.5^{\mathrm{A}}$ & $18.8 \pm 3.8^{\mathrm{A}}$ \\
D-dimer $(\mathrm{ng} / \mathrm{ml})$ & $647.7 \pm 79.1$ & $469.8 \pm 61.2$ & $950.5 \pm 52.5^{\mathrm{B}}$ & $873.2 \pm 130.0^{\mathrm{A}}$ \\
PF4 $(\mathrm{ng} / \mathrm{ml})$ & $461.6 \pm 19.2$ & $350.0 \pm 12.0^{\mathrm{C}}$ & $455.7 \pm 8.3$ & $469.0 \pm 18.7$
\end{tabular}

For TAT, $n=4$ and 12 per genotype for CD and HFD, respectively. For D-dimer and PF4, $n=4$ and $n=8$ per genotype for CD and HFD, respectively. Data are presented as the mean \pm SEM and analyzed by ANOVA with StudentNewman Keuls post hoc test. ${ }^{A} P<0.05,{ }^{B} P<0.01$ for $C D$ vs. HFD of the same genotype; ${ }^{\mathrm{C}} P<0.05$ for WT vs. Fib ${ }^{390-396 A}$ on the same diet. 
A

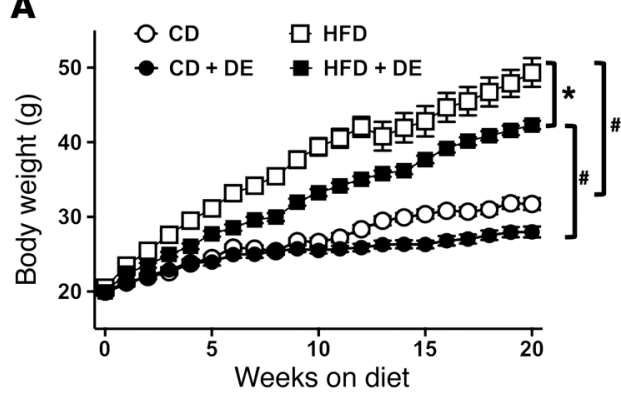

B

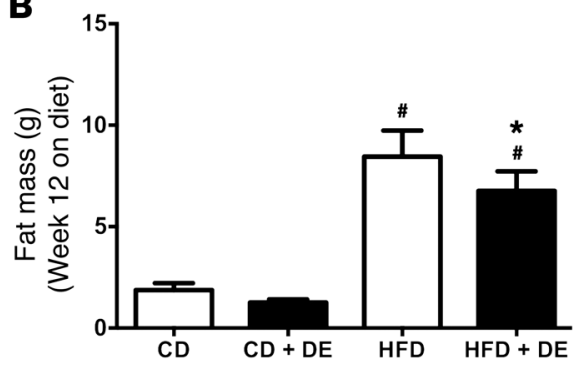

C

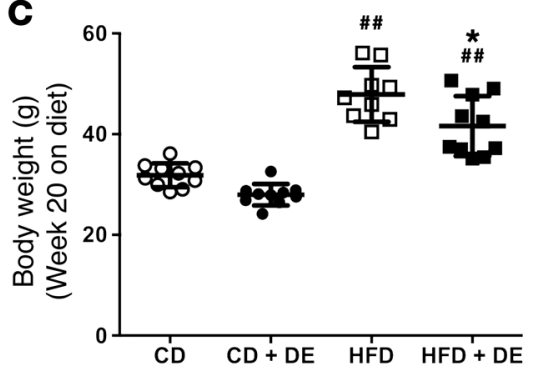

D Plasma clot

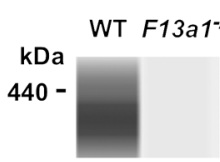
kDa
$440-$ $C D$

Extracted mouse eWAT

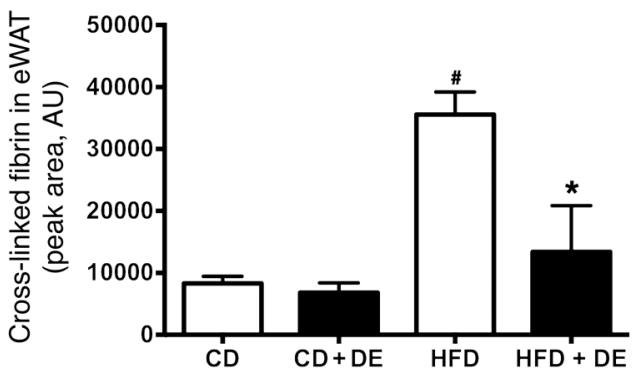

$\mathbf{E}$
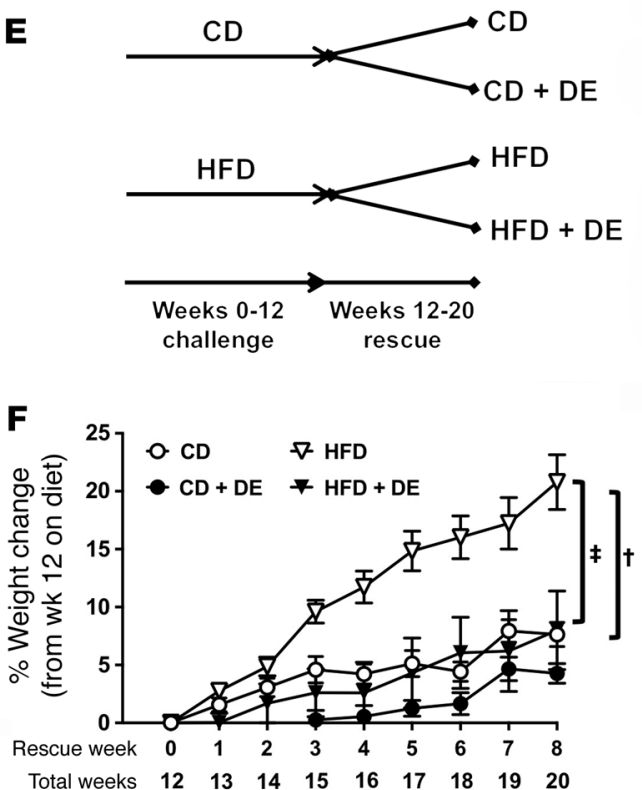

Figure 9. Treatment of mice with DE protects against the development of HFD-driven obesity and limits progression of established obesity. (A-C) Mice were fed a CD, a CD formulated with the direct thrombin inhibitor dabigatran etexilate (DE, $7.5 \mathrm{mg} / \mathrm{g})$, a 60\% HFD, or a 60\% HFD with DE $(7.5 \mathrm{mg} / \mathrm{g})(n=9-10 \mathrm{mice}$ per group). (A) Mean body weights of mice over a 20-week feeding period. (B) Body mass composition analysis for fat mass at week 12 on diet. (C) Distribution of body weights at week 20 on diet. (D) Fibrin(ogen) species were detected in clots formed in vitro with plasma from WT and F13a1 $1^{1-}$ mice using a capillary Western blot approach. Levels of fibrin(ogen) species were also detected in extracts of eWAT from 20-week CD-and HFD-fed control and DE-treated mice. For quantification, eWAT was used from $n=3-4$ mice for CD and $n=7$ for HFD. (E and F) WT mice were fed a CD or a 60\% HFD for 12 weeks, after which the mice received the same diet for an additional 8 weeks but were randomized to receive diet with or without DE. (E) Summary of experimental design strategy and feeding schedule for the DE rescue experiment. (F) Change in body weight over time for the 8 weeks of DE treatment. Data are expressed as the mean \pm SEM. Data were analyzed by 2-way ANOVA with Student-Newman-Keuls post hoc test. ${ }^{*} P<0.05$ for analyses comparing differences between HFD and HFD+DE. ${ }^{\#} P<0.05,{ }^{\#} P<0.01$ for analyses comparing differences between HFD and CD or between HFD+DE and CD+DE. For the DE intervention study, ${ }^{\dagger} P<0.05$ for mice fed a HFD versus $C D$ (neither with DE); ${ }^{\ddagger} P<0.05$ for mice on the same diet not treated with DE.

cifically associated with the most detrimental manifestations of NAFLD (data not shown) (34). In addition, DE treatment of HFDfed mice provided a significant protection from HFD-induced increase in blood glucose levels (Table 3). Collectively, these studies are the first, to our knowledge, to demonstrate that intervention with a direct thrombin inhibitor attenuates the progression of HFD-induced obesity and its associated pathologies.

\section{Discussion}

A key objective of our studies presented here was to determine whether fibrinogen promotes exacerbation of HFD-induced obesity, adipose tissue inflammation, and the downstream development of metabolic sequelae. Supporting a functional role for fibrin deposits are previous findings indicating extravascular fibrin- (ogen) is a potent proinflammatory disease modifier of inflammatory arthritis, neuroinflammatory disease, muscular dystrophy, and colitis (35-38). Here, we show evidence of extensive fibrin(ogen) deposition in both WAT and liver from obese mice that was absent in nonobese mice. These fibrin(ogen) deposits appeared to serve as foci for the accumulation of macrophages. Importantly, a virtually identical fibrin(ogen) staining pattern was observed in adipose tissue from obese patients, providing direct evidence in support of a role for this provisional matrix protein in human obesity-related disease.

Fibrin(ogen) deposits in tissues may influence the obese phenotype through several non-mutually exclusive mechanisms. However, our data suggest that a key mechanism of protection from HFD-induced obesity observed in Fib $\gamma^{390-396 A}$ mice is down- 
Table 2. Summary of body weight, body composition, hepatocellular injury (ALT), and glucose for mice fed a CD or HFD for 12 weeks

Diet:

CD

$\begin{array}{lc}\text { Body weight }(\mathrm{g}) & 28.0 \pm 0.7 \\ \text { Fat mass }(\%) & 5.8 \pm 0.8 \\ \text { Lean mass }(\%) & 63.3 \pm 0.6 \\ \text { ALT }(\mathrm{U} / \mathrm{l}) & 50.1 \pm 6.1 \\ \text { Clucose }(\mathrm{mg} / \mathrm{dl}) & 165.3 \pm 7.9 \\ n & 18\end{array}$

Data are presented as the mean \pm SEM and analyzed by Student's $t$ test; ${ }^{A} P<0.05$.

regulation of adipose inflammation and ATM function. This hypothesis is supported by multiple previous studies. In 2 separate reports, the elimination of macrophages in mice resulted in significant protection from HFD-induced weight gain and adiposity analogous to the protection from obesity we observed in Fib $\gamma^{390-396 A}$ mice $(29,30)$. The obesity phenotype is specifically associated with accumulation and activation of M1-polarized proinflammatory macrophages, whereas M2-polarized antiinflammatory macrophages are associated with a lean phenotype and protection from obesity $(31,39)$. It is possible that adipose fibrin(ogen) functions through the $\gamma^{390-396}$ motif to drive ATMs to the obese M1 phenotype. Our gene expression analyses indicating reduced $\operatorname{Tnf}-\alpha$ and Ccl 2 mRNA expression in WAT from HFD-challenged Fib $\gamma^{390-396 A}$ mice are consistent with this concept. Fibrin(ogen) $\gamma^{390-396 \mathrm{~A}}$ may alter obesity by failing to stimulate M1 polarization or by actively promoting a protective M2 polarization in adipose. Although additional investigation is required to define the precise impact of fibrin(ogen) on macrophage activation in obesity, it is notable that we did not observe higher levels of M2 markers (e.g., Arg1) in WAT from HFD-challenged Fib $\gamma^{390-396 A}$ mice (data not shown), suggesting that fibrin(ogen) $\gamma^{390-396 \mathrm{~A}}$ expression results in a failure of M1 polarization, rather than an active shift toward an M2 phenotype.

A central question addressed by the current study was how fibrin(ogen) modifies the local expression of soluble mediators that influence the obesity phenotype. Notably, the genetic elimination or pharmacological depletion of either TNF- $\alpha$ or MCP- 1 results in significant protection from HFD-induced insulin resistance and $\operatorname{NAFLD}(24,26,40)$. We observed significantly reduced local expression of Tnf- $\alpha$ mRNA levels in HFD-challenged Fib $\gamma^{390-396 A}$ mice that tracked with protection from the development of glucose dysmetabolism and NAFLD. We postulate that the reduction in obesity sequelae is a function of diminished body weight and adiposity in HFD-fed Fibr ${ }^{390-396 A}$ mice. However, the protection from HFD-induced NAFLD may be multifactorial, a product of reduced weight gain and reduced local fibrin-driven inflammation in the liver. Notably, the single loss of either TNF- $\alpha$ or MCP1 does not confer protection from weight gain and increased adiposity $(24,26,40)$. This suggests that fibrin(ogen) alters the function of adipocytes or preadipocytes (a) through a requisite combination of soluble mediators, (b) through ATM-mediated mechanisms beyond the production of proinflammatory mediators, (c) by directly engaging adipocytes or preadipocytes, or (d) by some combination of these mechanisms. Diminished $\mathrm{F} 4 / 80^{+}$ macrophages were observed in the eWAT of HFD-fed Fib $\gamma^{390-396 A}$ mice, which was likely a product of overall reduced inflammation in these animals. In regard to adipocyte function, it is notable that when adipose tissue expands as a consequence of HFD feeding, preadipocytes are programmed to differentiate, permitting a hyperplastic expansion of functional adipocytes that preserves insulin sensitivity. Adipose infiltration by ATMs has been shown to promote an adipogenic deficit by suppressing preadipocyte differentiation $(41,42)$. It is possible that WAT fibrin(ogen) deposits promote both production of key proinflammatory soluble mediators that drive obesity sequelae and ATM-mediated suppression of preadipocyte differentiation.

Our studies directly implicate the $\gamma^{390-396}$ motif in fibrin(ogen)driven HFD-induced obesity. This motif supports binding to the leukocyte integrin receptor $\alpha_{\mathrm{M}} \beta_{2}$. A recent study documented significantly diminished adipose tissue inflammation as well as protection from HFD-induced insulin resistance and fatty liver disease in $\alpha_{\mathrm{M}}$-deficient mice, results that are complementary to our data (43). Intriguingly, HFD-fed $\alpha_{M}$-deficient mice did not show protection from weight gain, suggesting either that various $\alpha_{M}$ ligands have opposing effects on HFD-induced WAT adiposity, or that the $\gamma^{390-396}$ motif promotes WAT weight gain through mechanisms separate from $\alpha_{\mathrm{M}^{*}}$. In this regard, the $\beta_{2}$-integrin family member $\alpha_{x} \beta_{2}$ (CD11c/CD18) has been shown to bind fibrinogen through the $\gamma^{390-396}$ domain and is expressed by monocytes/ macrophages $(44,45)$. Inflammatory CD11 $\mathrm{c}^{+}$cells are significantly increased in obese adipose tissue and appear to contribute to the obese phenotype (46-48).

The coagulation transglutaminase factor XIII also binds the fibrinogen $\gamma^{390-396}$ motif (23). F13a1 gene expression is significantly upregulated in WAT of both obese patients and mice fed a HFD $(49,50)$. Further, factor XIII has been suggested to act as a negative regulator of adipogenesis (50). Nevertheless, we show that loss of factor XIII activity does not phenocopy results observed in Fib $\gamma^{390-396 A}$ mice, as $\mathrm{F} 13 \mathrm{al}^{-/-}$mice gain the same amount of weight as WT mice following HFD feeding. Collectively these findings suggest that the primary effect of the $\gamma^{390-396 \mathrm{~A}}$ mutation in obesi-

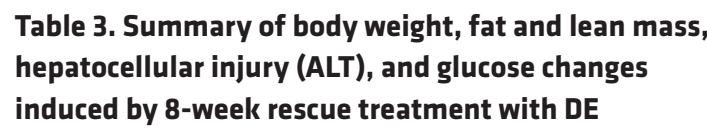

Table 3. Summary of body weight, fat and lean mass, hepatocellular injury (ALT), and glucose changes induced by 8 -week rescue treatment with DE

\section{Impact of DE treatment (12-20 weeks)}

Diet:

CD

HFD

$\begin{array}{lcccc}\text { Rescue: } & - & \mathrm{DE} & - & \mathrm{DE} \\ \Delta \text { Body weight }(\mathrm{g}) & 1.5 \pm 0.3^{\mathrm{A}} & 0.8 \pm 0.2 & 7.9 \pm 1.0^{\mathrm{A}, \mathrm{B}} & 3.8 \pm 1.5^{\mathrm{A}, \mathrm{C}} \\ \Delta \text { Fat mass }(\%) & 15.1 \pm 8.7 & 8.9 \pm 20.9 & 35.8 \pm 11.4^{\mathrm{A}, \mathrm{B}} & 6.9 \pm 5.2 \\ \Delta \text { Lean mass }(\%) & -0.5 \pm 1.0 & 1.9 \pm 1.2 & 2.9 \pm 0.9^{\mathrm{A}, \mathrm{B}} & 3.0 \pm 0.8^{\mathrm{A}} \\ \Delta \text { ALT }(\mathrm{U} / \mathrm{l}) & -38.5 \pm 8.5^{\mathrm{A}} & -9.6 \pm 3.1 & 67.2 \pm 28.77^{\mathrm{A}, \mathrm{B}} & 6.6 \pm 13.7^{\mathrm{C}} \\ \Delta \text { Glucose }(\mathrm{mg} / \mathrm{dl}) & 17.3 \pm 14.0 & -8.4 \pm 9.7 & 36.8 \pm 10.4^{\mathrm{A}} & -11.0 \pm 14.2^{\mathrm{C}} \\ N & 10 & 8 & 9 & 9\end{array}$

Data are presented as the mean \pm SEM and analyzed by ANOVA with Student-Newman Keuls post hoc test. ${ }^{A} P<0.05$ for change in parameter at 20 vs. 12 weeks; ${ }^{B} P<0.05$ for mice fed a HFD vs. CD (neither with DE); and ${ }^{c} P<0.05$ for mice on the same diet treated vs. not treated with $D E$ 
ty is not mediated simply by a decrease in factor XIII binding. A recent study by Myneni and colleagues indicated that HFD-fed $\mathrm{F} 3 \mathrm{al}^{-/-}$mice gain the same amount of weight as WT mice, aligned with our findings, but that $F 13 \mathrm{a1}^{-/-}$mice had diminished adipose tissue inflammation and were protected from insulin resistance (51). It was suggested that the mechanism of factor XIII exacerbation of adipose inflammation and obesity sequelae was linked to fibronectin (51); however, a possible link to fibrin(ogen) was not considered. We postulate that factor XIII may be influencing HFD-induced pathologies through a mechanism whereby crosslinked fibrin drives a greater proinflammatory response and has a greater impact on ATM function than non-cross-linked fibrin, a general hypothesis supported by previous work in our laboratory (52). However, defining the precise interplay of fibrin(ogen) and factor XIII in obesity sequelae, including insulin resistance and NAFLD, remains for future investigation.

The identification of fibrinogen as a potent modifier of obesity implicates both upstream coagulation system components and downstream fibrinolytic factors as potential obesity modifiers. In this regard, elevated circulating levels of tissue factor (TF) and factor VII have been documented in obese patients with T2D and in mice fed a HFD $(9,10)$. High levels of bloodborne $\mathrm{TF}$ are considered a biomarker of severe microvascular disease in T2D patients $(53,54)$. Further, $T F$ mRNA levels and activity are increased in the visceral adipose tissue of obese mice; specifically, TF expression is high in infiltrating ATMs (55, 56). Direct evidence of a causative relationship for TF in obesity was shown in mice lacking the cytoplasmic tail of TF, which conferred protection from HFD-induced weight gain and insulin resistance that was linked to $\mathrm{TF} /$ factor VIIa-mediated signaling by PAR2 in ATMs (57). Additionally, mice with low TF expression in the bone marrow compartment similarly were protected from HFD-induced weight gain (58). Here, we provide strong evidence that the TF/thrombin/fibrin(ogen) pathway also promotes obesity, glucose dysmetabolism, and fatty liver disease. Fibrinolytic/plasminogen activation system components have long been associated with obesity and metabolic syndrome. In particular, plasminogen activator inhibitor-1 (PAI-1) is markedly elevated in obese patients and is considered a biomarker of obesity and metabolic syndrome (59-62). Visceral adipose tissue is the largest source of PAI- 1 in obese patients and is derived from both adipocytes and infiltrating ATMs (62-65). The genetic elimination of PAI-1 in mice fed a HFD has been shown to result in diminished obesity and insulin resistance in several studies (66-69). In addition, mice deficient in tissue plasminogen activator (tPA) were shown to develop exacerbated obesity following HFD challenge (70). Collectively these findings are all consistent with the concept that persistent fibrin deposits drive diet-induced obesity.

Analyses of Thbdro mice indicated that these animals develop exacerbated HFD-induced obesity and associated sequelae. Although it is possible that the increased obesity observed in these animals was secondary to loss of Thbd antiinflammatory mechanisms, our data are consistent with our prevailing hypothesis that it is exacerbated thrombin procoagulant function driving HFD-induced obesity in these mice. Indeed, our finding that therapeutic DE administration effectively halts the progression of obesity-associated sequelae in mice suggests that targeting thrombin may be a beneficial therapeutic approach. Previous studies showed that intervention with another thrombin inhibitor, argatroban, significantly reduced systemic inflammation and plasma cholesterol levels in mice with established hypercholesterolemia and obesity (71). Could the novel oral anticoagulants (NOACs), currently relegated to antithrombotic therapy, be therapeutically beneficial in patients with established obesity? Obesity and its sequelae often coexist with atrial fibrillation, a primary indication for NOAC therapy (72). Notably, weight loss itself has been shown to reduce stroke risk associated with atrial fibrillation (73). Thus, NOAC therapy in patients with an existing indication may confer an additional metabolic benefit. Although the treatment strategy is anchored in strong and reproducible experimental results, the idea of treating patients with metabolic syndrome and obesity with NOACs may be viewed as challenging. However, the overall favorable bleeding risk profile of these agents, together with the emergence of highly specific and efficacious reversal agents for available NOACs, may ultimately tip the risk/benefit balance to favor this possibility.

In this study, we define a novel mechanism of inflammation in experimental obesity mediated by the fibrin(ogen) leukocyte integrin $\alpha_{M} \beta_{2}$-binding motif. Through gain- and loss-of-function studies targeting thrombin generation and downstream substrates, these studies are, to our knowledge, the first to ascribe thrombin-driven obesity to a function of fibrin(ogen) and not PAR1 signaling. Moreover, to our knowledge, these studies are the first to experimentally demonstrate that procoagulant mutations are capable of exacerbating obesity-associated pathologies, authenticating association studies in humans $(16,17,74)$.

The deciphered mechanism is responsive to modulation with thrombin-targeted FDA-approved therapeutic agents. More specifically, fibrin-driven obesity appears amenable to targeted inhibition of the fibrin(ogen) $\gamma^{390-396}$ motif. In summary, the thrombin/ fibrin(ogen) axis is not only a critical trigger of HFD-mediated systemic inflammation, but a pharmacologically and genetically adjustable driver of obesity and related pathologies such as NAFLD and glucose dysmetabolism.

\section{Methods}

Mice. Thbd ${ }^{\text {Pro }}$, Fgg 390-396A (Fib $\gamma^{390-396 A}$ ), Fgg 45 (Fib $\left.\gamma^{45}\right), F 13 a 1^{-1-}$, and $\mathrm{F} 2 \mathrm{r}^{-/}$mice backcrossed 7 generations to $\mathrm{C} 57 \mathrm{BL} / 6$ were all previously described (20, 21, 75-77). Age-matched littermates of WT and homozygous mutant male mice between the ages of 8 and 12 weeks were used. For DE experiments, male C57BL/6J mice at 6 weeks of age were purchased from the Jackson Laboratory and allowed to acclimate in the animal facility for 2 weeks before being enrolled in studies. Mice were housed at an ambient temperature of $22^{\circ} \mathrm{C}$ with alternating 12-hour light/12-hour dark cycles, and provided water and rodent chow ad libitum before study initiation.

HFD-induced obesity. Mice were fed ad libitum a CD of standard mouse chow that provides about $13 \%$ of the kilocalories (kcal) derived from fat (Laboratory Autoclavable Rodent Diet 5010; LabDiet) or a HFD with $60 \%$ of the kcal derived from fat (catalog D12492; Research Diets Inc.). In some experiments, mice were fed a CD with $10 \%$ of the kcal derived from fat (catalog 56834; Test Diet) or a HFD with $45 \%$ of the kcal derived from fat (catalog 55629; Test Diet). In DE studies, mice 
were fed a 60\% HFD (catalog 112252; Dyets Inc.) identical to catalog D12492 from Research Diets Inc. with or without $7.5 \mathrm{mg} / \mathrm{g}$ DE. Control mice were fed modified AIN-93M CD (Dyets Inc., 10\% of kcal derived from fat) with or without $7.5 \mathrm{mg} / \mathrm{g} \mathrm{DE}$. Both the HFD and the CD with and without $\mathrm{DE}$ were formulated to contain $1 \%$ peanut flavoring to mask the bitter taste of the drug. Note that terminal circulating DE concentrations were determined for each group and found to be 388.2 and $248.0 \mathrm{ng} / \mathrm{ml}$ for the CD and HFD groups, respectively, in the prophylactic study and 327.0 and $358.2 \mathrm{ng} / \mathrm{ml}$ for the CD and HFD groups, respectively, in the rescue study. The total body weight of mice and weight of food consumed were determined weekly. Whole body composition analysis was determined as indicated using a 3-in-1 analyzer for fat mass, lean mass, and water (EchoMRI or Bruker NMR). At the end of the observation period, mice were euthanized for collection of citrate plasma, serum, and organ harvest. The weight of eWAT, iWAT, BAT, and liver was determined at harvest.

Histopathology and clinical chemistry. Mouse eWAT and a medial or left lateral lobe of the liver were used for histological analysis. Biopsy samples of human visceral adipose and liver were obtained from the Cincinnati Children's Hospital Pediatric Obesity Tissue Repository from obese patients with either normal liver histology or a histologically confirmed diagnosis of NASH. Tissue sections of visceral adipose from nonobese individuals were obtained from Origene. Formalin-fixed tissue sections $(5 \mu \mathrm{m})$ were stained with $\mathrm{H} \& \mathrm{E}$ or by immunohistochemistry with either a rabbit polyclonal anti-mouse fibrinogen antibody (previously described in ref. 36) or a monoclonal anti-human fibrinogen antibody (reference A0080; Dako). Fibrin(ogen) staining was quantified using Image J (NIH). Epididymal adipose sections stained with F4/80 (catalog MCA497G; Bio-Rad Laboratories) were scanned and digitized using a Virtual Slide System VS110 (Olympus). The area of individual adipocytes was calculated using Olympus cellSens Dimension Desktop Software (version 1.15). Specifically, 50 individual adipocytes selected from at least 5 different areas of the digitized sections were analyzed per sample.

For genetically modified mice, blood was collected at the end of the observation period from the inferior vena cava in citrate. For studies where mice were treated with DE-containing chow, blood was collected by retro-orbital bleeding after 12 weeks on CD or HFD and analyzed for serum alanine aminotransferase (ALT) and glucose levels. ELISA analysis was used to determine the plasma levels of TAT complexes (Siemens Enzygnost TAT kit), D-dimer (Biomatik Mouse D-dimer kit), and PF4 (RayBio Mouse PF-4 ELISA kit). Serum ALT activity was determined using a commercially available reagent (Thermo Fisher Scientific). Plasma cytokines, chemokines, and adipokines were determined using Milliplex Magnetic Bead multiplex or single-plex kits (Millipore) and read using a Bio-Plex (Bio-Rad Laboratories). Concentrations were calculated from standard curves using recombinant proteins and expressed in picograms per milliliter or micrograms per milliliter.

For analysis of blood glucose, mice were fasted for 6 hours followed by blood glucose measurements taken using an ACCU-check Performa meter (Roche) from a tail vein blood sample. Glucose tolerance tests were performed after 16 weeks on diet. Mice were fasted for 6 hours, and dextrose was administered by i.p. injection at a dose of $1 \mathrm{~g} / \mathrm{kg}$ mouse body weight with blood glucose readings taken at 15-minute intervals. Insulin tolerance tests were performed on mice after 18 weeks on diet. Human recombinant insulin (Novolin-R; Novo Nordisk) was administered by i.p. injection at a dose of $0.75 \mathrm{U} / \mathrm{kg}$ body weight. Blood glucose readings were taken at 15-minute intervals. For both glucose and insulin tolerance tests, AUC was determined for blood glucose profiles from individual mice.

Hepatic triglyceride determination. Hepatic triglyceride content was determined as described previously (78). Briefly, $100 \mathrm{mg}$ of frozen liver tissue was homogenized, mixed with chloroform/methanol (2:1), and incubated overnight at room temperature. Subsequently, $\mathrm{H}_{2} \mathrm{O}$ was added with collection of the lower lipid phase by centrifugation and vacuum concentration. The lipid pellets were dissolved in a mixture of isopropanol and Triton X-100 and analyzed spectrophotometrically for triglycerides $(600 \mathrm{~nm})$ using commercially available reagents (Pointe Scientific).

RNA isolation, cDNA synthesis, and real-time PCR. Total RNA was isolated from $100 \mathrm{mg}$ of tissue using Trizol (Ambion) according to the manufacturer's protocol. RNA $(1 \mu \mathrm{g})$ was used for cDNA synthesis with a High Capacity cDNA Reverse Transcription kit (Applied Biosystems). Levels of TNF- $\alpha$ (Tnf), MCP-1 (Ccl2), F4/80 (Adgre1), PPAR $\gamma$ (Pparg), CIDEA (Cidea), CD36 (Cd36), UCP1 (Ucp1), UCP2 $(U c p 2)$, and $\beta_{2}$-microglobulin (B2m) were determined using TaqMan gene expression assays (Applied Biosystems) on an ABI StepOne Plus sequence detection system (Applied Biosystems). The expression of each gene was normalized relative to $B 2 m$ expression levels, and relative expression level determined using the Pfaffl method (79).

Detection of fibrin(ogen) by capillary Western blotting. To assess WAT levels of cross-linked high-molecular weight fibrin(ogen), 100 $\mathrm{mg}$ of eWAT was homogenized in $8 \mathrm{M}$ urea, $40 \mathrm{mM} \mathrm{DTT}, 12.5 \mathrm{mM}$ EDTA based on protocols outlined previously $(23,80)$, rotated end over end in a $60^{\circ} \mathrm{C}$ incubator for 2 hours, and incubated stationary at $60^{\circ} \mathrm{C}$ overnight ( 15 hours). Samples were collected by centrifugation and resolved on Wes 66- to $440-\mathrm{kDa} 25$-microcapillary gels (Protein Simple). Fibrin(ogen) was detected with polyclonal rabbit anti-human fibrinogen antibody (reference A0080; Dako) per the manufacturer's protocol (Protein Simple). To identify precise species, in vitro fibrin polymerization was performed using citrated WT and $\mathrm{F}_{13 \mathrm{a1}} \mathrm{1}^{--}$mouse plasma, stimulated with human $\alpha$-thrombin (2 $\mathrm{U} / \mathrm{ml}$ final) (Enzyme Research Laboratories) in a $\mathrm{CaCl}_{2}(10$ $\mathrm{mM}$ final) buffer for 30 minutes at $37^{\circ} \mathrm{C}$. Samples were processed in urea/DTT/EDTA buffer, and fibrin(ogen) species were detected using capillary Western blot.

Intestinal fat absorption. Mice were fed with a CD or $60 \%$ HFD for 16 weeks, after which a diet containing $5 \%$ sucrose polybehenate, a nonabsorbable food additive, was fed to the mice ad libitum for 3 days. Fecal pellets were collected on days 3 and 4 . Fat absorption was calculated from the ratios of behenic acid to other fatty acids in the diet and in the feces by gas chromatography of fatty acid methyl esters.

Statistics. In studies in which the weights of mice over time were determined, the data were analyzed by repeated-measures ANOVA. Comparison of 2 groups was performed using a 2-tailed Student's $t$ test. Comparison of 3 or more groups was performed using 1-way or 2-way ANOVA and Student-Newman-Keuls post hoc test. $P$ values for all comparisons are indicated in the figure legends, but for all analyses the criterion for statistical significance was $P$ less than 0.05.

Study approval. Mice were maintained in Association for Assessment and Accreditation of Laboratory Animal Care International-accredited facilities and approved by the IACUC at Cincinnati Children's Hospital 
Research Foundation or the IACUC at Michigan State University. The Cincinnati Children's Hospital Medical Center IRB approved studies involving the analysis of human tissue samples. Deidentified samples were obtained through the Pediatric Obesity Tissue Repository at the Cincinnati Children's Hospital Medical Center.

\section{Author contributions}

AKK, SRA, SD, JPL, and MJF designed the study, performed experiments and data analysis, and participated in the writing of the manuscript. ST, JSP, ESM, HW, APO, and NM were involved with conception of the project, data analysis, and writing of the manuscript. AG and JvR provided DE and assisted with conception of the project and editing of the manuscript. All authors contributed to final editing of the manuscript.

\section{Acknowledgments}

We thank Carolina Cruz, Alice Jone, Cheryl Rewerts, Traci Stankiewicz, and Maureen Shaw for their excellent technical assistance. This work was supported by a research contract from Boehringer Ingelheim (to JPL), grants DK099222 (to SD), ES017537 (to JPL), and HL112603 (to MJF) from the NIH, and a Pilot and Feasibility grant (to MJF) through NIH grant P30 DK078392 of the Digestive Diseases Research Core Center at Cincinnati Children's Hospital Medical Center.

Address correspondence to: Matthew J. Flick, Division of Experimental Hematology and Cancer Biology, Cincinnati Children's Hospital, 3333 Burnet Avenue, Cincinnati, Ohio 45229-3039, USA. Phone: 513.636.6628; Email: matthew.flick@cchmc.org.
1. Li C, et al. Surveillance of certain health behaviors and conditions among states and selected local areas - Behavioral Risk Factor Surveillance System, United States, 2009. MMWR Surveill Summ. 2011;60(9):1-250.

2. Hotamisligil GS. Inflammation and metabolic disorders. Nature. 2006;444(7121):860-867.

3. Cao H, et al. Regulation of metabolic responses by adipocyte/macrophage fatty acid-binding proteins in leptin-deficient mice. Diabetes. 2006;55(7):1915-1922.

4. Neels JG, Pandey M, Hotamisligil GS, Samad F. Autoamplification of tumor necrosis factor- $\alpha$ : a potential mechanism for the maintenance of elevated tumor necrosis factor- $\alpha$ in male but not female obese mice. Am J Pathol. 2006;168(2):435-444.

5. Carolan E, et al. The impact of childhood obesity on inflammation, innate immune cell frequency, and metabolic microRNA expression. J Clin Endocrinol Metab. 2014;99(3):E474-E478.

6. Cao H. Adipocytokines in obesity and metabolic disease. JEndocrinol. 2014;220(2):T47-T59.

7. Gregor MF, Hotamisligil GS. Inflammatory mechanisms in obesity. Annu Rev Immunol. 2011;29:415-445.

8. Finucane MM, et al. National, regional, and global trends in body-mass index since 1980: systematic analysis of health examination surveys and epidemiological studies with 960 country-years and $9 \cdot 1$ million participants. Lancet. 2011;377(9765):557-567.

9. Morange PE, Alessi MC. Thrombosis in central obesity and metabolic syndrome: mechanisms and epidemiology. Thromb Haemost. 2013;110(4):669-680.

10. Kahn SE, Hull RL, Utzschneider KM. Mechanisms linking obesity to insulin resistance and type 2 diabetes. Nature. 2006;444(7121):840-846.

11. Kopp CW, et al. Weight loss reduces tissue factor in morbidly obese patients. Obes Res. 2003;11(8):950-956.

12. Christiansen SC, et al. The relationship between body mass index, activated protein $\mathrm{C}$ resistance and risk of venous thrombosis. J Thromb Haemost. 2012;10(9):1761-1767.

13. Klovaite J, Benn M, Nordestgaard BG. Obesity as a causal risk factor for deep venous thrombosis: a Mendelian randomization study. J Intern Med. 2015;277(5):573-584.
14. Dehlendorff C, Andersen KK, Olsen TS. Body mass index and death by stroke: no obesity paradox. JAMA Neurol. 2014;71(8):978-984.

15. Stalls CM, Triplette MA, Viera AJ, Pathman DE, Cohen MG, Rossi JS. The association between body mass index and coronary artery disease severity: a comparison of black and white patients. Am Heart J. 2014;167(4):514-520.

16. de Moerloose P, Wutschert R, Heinzmann M, Perneger T, Reber G, Bounameaux H. Superficial vein thrombosis of lower limbs: influence of factor V Leiden, factor II G20210A and overweight. Thromb Haemost. 1998;80(2):239-241.

17. Avellone G, et al. Effects of heparin treatment on hemostatic abnormalities in obese non-insulin-dependent diabetic patients. Metab Clin Exp. 1997;46(8):930-934.

18. Papatheodoridis GV, et al. Thrombotic risk factors and liver histologic lesions in non-alcoholic fatty liver disease. J Hepatol. 2009;51(5):931-938.

19. Murano I, et al. Dead adipocytes, detected as crown-like structures, are prevalent in visceral fat depots of genetically obese mice. JLipid Res. 2008;49(7):1562-1568.

20. Flick MJ, et al. Leukocyte engagement of fibrin(ogen) via the integrin receptor alphaMbeta2/Mac-1 is critical for host inflammatory response in vivo. JClin Invest. 2004;113(11):1596-1606.

21. Holmbäck K, Danton MJ, Suh TT, Daugherty CC, Degen JL. Impaired platelet aggregation and sustained bleeding in mice lacking the fibrinogen motif bound by integrin $\alpha \operatorname{IIb} \beta 3$. EMBO J. 1996;15(21):5760-5771.

22. Mao J, et al. Overnutrition stimulates intestinal epithelium proliferation through $\beta$-catenin signaling in obese mice. Diabetes. 2013;62(11):3736-3746.

23. Aleman MM, et al. Factor XIII activity mediates red blood cell retention in venous thrombi. J Clin Invest. 2014;124(8):3590-3600.

24 . De Taeye BM, et al. Macrophage TNF- $\alpha$ contributes to insulin resistance and hepatic steatosis in diet-induced obesity. Am J Physiol Endocrinol Metab. 2007;293(3):E713-E725.

25. Endo M, Masaki T, Seike M, Yoshimatsu H. TNF$\alpha$ induces hepatic steatosis in mice by enhancing gene expression of sterol regulatory element binding protein-1c (SREBP-1c). Exp Biol Med (Maywood). 2007;232(5):614-621.

26. Kanda H, et al. MCP-1 contributes to macrophage infiltration into adipose tissue, insulin resistance, and hepatic steatosis in obesity. JClin Invest. 2006;116(6):1494-1505.

27. Takahashi K, et al. Adiposity elevates plasma MCP-1 levels leading to the increased CD11b-positive monocytes in mice. J Biol Chem. 2003;278(47):46654-46660.

28. Harley IT, et al. IL-17 signaling accelerates the progression of nonalcoholic fatty liver disease in mice. Hepatology. 2014;59(5):1830-1839.

29. Bu L, Gao M, Qu S, Liu D. Intraperitoneal injection of clodronate liposomes eliminates visceral adipose macrophages and blocks high-fat dietinduced weight gain and development of insulin resistance. AAPS J. 2013;15(4):1001-1011.

30. Lee B, Qiao L, Kinney B, Feng GS, Shao J. Macrophage depletion disrupts immune balance and energy homeostasis. PLoS One. 2014;9(6):e99575.

31. Kitade $\mathrm{H}$, et al. CCR5 plays a critical role in obesity-induced adipose tissue inflammation and insulin resistance by regulating both macrophage recruitment and M1/M2 status. Diabetes. 2012;61(7):1680-1690.

32. Kassel KM, et al. Protease-activated receptor 1 and hematopoietic cell tissue factor are required for hepatic steatosis in mice fed a Western diet. Am J Pathol. 2011;179(5):2278-2289.

33. Kopec AK, et al. Thrombin inhibition with dabigatran protects against high-fat diet-induced fatty liver disease in mice.J Pharmacol Exp Ther. 2014;351(2):288-297.

34. Tandra S, et al. Presence and significance of microvesicular steatosis in nonalcoholic fatty liver disease.J Hepatol. 2011;55(3):654-659.

35. Adams RA, et al. The fibrin-derived gamma377395 peptide inhibits microglia activation and suppresses relapsing paralysis in central nervous system autoimmune disease. J Exp Med. 2007;204(3):571-582.

36. Flick MJ, et al. Fibrin(ogen) exacerbates inflammatory joint disease through a mechanism linked to the integrin alpha(M)beta(2) binding motif. JClin Invest. 2007;117(11):3224-3235.

37. Steinbrecher KA, et al. Colitis-associated cancer is dependent on the interplay between the hemostatic and inflammatory systems and supported by integrin alpha(M)beta(2) engagement of fibrinogen. Cancer Res. 2010;70(7):2634-2643. 
38. Vidal B, et al. Fibrinogen drives dystrophic muscle fibrosis via a TGFbeta/alternative macrophage activation pathway. Genes Dev. 2008;22(13):1747-1752.

39. Ji Y, et al. Activation of natural killer $\mathrm{T}$ cells promotes M2 Macrophage polarization in adipose tissue and improves systemic glucose tolerance via interleukin-4 (IL-4)/STAT6 protein signaling axis in obesity. J Biol Chem. 2012;287(17):13561-13571.

40. Li Z, et al. Probiotics and antibodies to TNF inhibit inflammatory activity and improve nonalcoholic fatty liver disease. Hepatology. 2003;37(2):343-350.

41. Lumeng CN, Bodzin JL, Saltiel AR. Obesity induces a phenotypic switch in adipose tissue macrophage polarization. JClin Invest. 2007;117(1):175-184.

42. Lumeng CN, Deyoung SM, Bodzin JL, Saltiel AR. Increased inflammatory properties of adipose tissue macrophages recruited during diet-induced obesity. Diabetes. 2007;56(1):16-23.

43. Wolf $\mathrm{D}$, et al. Inflammation, but not recruitment, of adipose tissue macrophages requires signalling through Mac-1 (CD11b/CD18) in diet-induced obesity (DIO). Thromb Haemost. 2017;117(2):325-338.

44. Ugarova TP, Yakubenko VP. Recognition of fibrinogen by leukocyte integrins. Ann N Y Acad Sci. 2001;936:368-385.

45. Sitrin RG, Pan PM, Srikanth S, Todd RF. Fibrinogen activates NF- $\kappa B$ transcription factors in mononuclear phagocytes. JImmunol. 1998;161(3):1462-1470.

46. Wu H, et al. CD11c expression in adipose tissue and blood and its role in diet-induced obesity. Arterioscler Thromb Vasc Biol.2010;30(2):186-192.

47. Wouters K, et al. Circulating classical monocytes are associated with $\mathrm{CD} 11 \mathrm{c}(+)$ macrophages in human visceral adipose tissue. Sci Rep. 2017;7:42665.

48. Stefanovic-Racic M, et al. Dendritic cells promote macrophage infiltration and comprise a substantial proportion of obesity-associated increases in $\mathrm{CD} 11 \mathrm{c}+$ cells in adipose tissue and liver. Diabetes. 2012;61(9):2330-2339.

49. Naukkarinen J, et al. Use of genome-wide expression data to mine the "Gray Zone" of GWA studies leads to novel candidate obesity genes. PLoS Genet. 2010;6(6):e1000976.

50. Myneni VD, Hitomi K, Kaartinen MT. Factor XIII-A transglutaminase acts as a switch between preadipocyte proliferation and differentiation. Blood. 2014;124(8):1344-1353.

51. Myneni VD, Mousa A, Kaartinen MT. Factor XIII-A transglutaminase deficient mice show signs of metabolically healthy obesity on high fat diet. Sci Rep. 2016;6:35574.

52. Raghu H, et al. Transglutaminase factor XIII promotes arthritis through mechanisms linked to inflammation and bone erosion. Blood. 2015;125(3):427-437.

53. Meerarani P, Moreno PR, Cimmino G, Badimon JJ. Atherothrombosis: role of tissue factor; link between diabetes, obesity and inflammation. Indian JExp Biol. 2007;45(1):103-110.

54. Buchs AE, Kornberg A, Zahavi M, Aharoni D, Zarfati C, Rapoport MJ. Increased expression of tissue factor and receptor for advanced glycation end products in peripheral blood mononuclear cells of patients with type 2 diabetes mellitus with vascular complications. Exp Diabesity Res. 2004;5(2):163-169.

55. Samad F, Pandey M, Loskutoff DJ. Regulation of tissue factor gene expression in obesity. Blood. 2001;98(12):3353-3358.

56. Samad F, Pandey M, Loskutoff DJ. Tissue factor gene expression in the adipose tissues of obese mice. Proc Natl Acad Sci U S A. 1998;95(13):7591-7596.

57. Badeanlou L, Furlan-Freguia C, Yang G, Ruf W, Samad F. Tissue factor-protease-activated receptor 2 signaling promotes diet-induced obesity and adipose inflammation. Nat Med. 2011;17(11):1490-1497.

58. Owens AP, et al. Monocyte tissue factor-dependent activation of coagulation in hypercholesterolemic mice and monkeys is inhibited by simvastatin. JClin Invest. 2012;122(2):558-568.

59. Kohler HP, Grant PJ. Plasminogen-activator inhibitor type 1 and coronary artery disease. N Engl JMed. 2000;342(24):1792-1801.

60. Alessi MC, Juhan-Vague I. Metabolic syndrome, haemostasis and thrombosis. Thromb Haemost. 2008;99(6):995-1000.

61. Mertens I, Verrijken A, Michiels JJ, Van der Planken M, Ruige JB, Van Gaal LF. Among inflammation and coagulation markers, PAI- 1 is a true component of the metabolic syndrome. Int JObes (Lond). 2006;30(8):1308-1314.

62. Alessi MC, Peiretti F, Morange P, Henry M, Nalbone G, Juhan-Vague I. Production of plasminogen activator inhibitor 1 by human adipose tissue: possible link between visceral fat accumulation and vascular disease. Diabetes. 1997;46(5):860-867.

63. Cigolini M, et al. Expression of plasminogen activator inhibitor-1 in human adipose tissue: a role for TNF- $\alpha$ ? Atherosclerosis. 1999;143(1):81-90.

64. Shimomura I, et al. Enhanced expression of PAI-1 in visceral fat: possible contributor to vascular disease in obesity. Nat Med.1996;2(7):800-803.

65. Bastelica D, et al. Stromal cells are the main plasminogen activator inhibitor-1-producing cells in human fat: evidence of differences between visceral and subcutaneous deposits. Arterioscler Thromb Vasc Biol. 2002;22(1):173-178.

66. Morange PE, Lijnen HR, Alessi MC, Kopp F, Collen D, Juhan-Vague I. Influence of PAI- 1 on adipose tissue growth and metabolic parameters in a murine model of diet-induced obesity. Arterioscler Thromb Vasc Biol. 2000;20(4):1150-1154.

67. Schäfer K, Fujisawa K, Konstantinides S, Loskutoff DJ. Disruption of the plasminogen activator inhibitor 1 gene reduces the adiposity and improves the metabolic profile of genetically obese and diabetic ob/ob mice. FASEB J. 2001;15(10):1840-1842.

68. Ma LJ, et al. Prevention of obesity and insulin resistance in mice lacking plasminogen activator inhibitor 1. Diabetes. 2004;53(2):336-346.

69. De Taeye BM, Novitskaya T, Gleaves L, Covington JW, Vaughan DE. Bone marrow plasminogen activator inhibitor-1 influences the development of obesity. J Biol Chem. 2006;281(43):32796-32805.

70. Morange PE, et al. Influence of t-pA and u-PA on adipose tissue development in a murine model of diet-induced obesity. Thromb Haemost. 2002;87(2):306-310.

71. Kassel KM, Sullivan BP, Cui W, Copple BL, Luyendyk JP. Therapeutic administration of the direct thrombin inhibitor argatroban reduces hepatic inflammation in mice with established fatty liver disease. Am J Pathol. 2012;181(4):1287-1295.

72. Pathak RK, Mahajan R, Lau DH, Sanders P. The implications of obesity for cardiac arrhythmia mechanisms and management. Can J Cardiol. 2015;31(2):203-210.

73. Pathak RK, et al. Long-Term Effect of Goal-Directed Weight Management in an Atrial Fibrillation Cohort: A Long-Term Follow-Up Study (LEGACY). J Am Coll Cardiol. 2015;65(20):2159-2169.

74. Papatheodoridis GV, et al. Thrombotic risk factors and liver histologic lesions in non-alcoholic fatty liver disease. J Hepatol. 2009;51(5):931-938.

75. Weiler-Guettler $\mathrm{H}$, et al. A targeted point mutation in thrombomodulin generates viable mice with a prethrombotic state. JClin Invest. 1998;101(9):1983-1991.

76. Palumbo JS, et al. Factor XIII transglutaminase supports hematogenous tumor cell metastasis through a mechanism dependent on natural killer cell function. J Thromb Haemost. 2008;6(5):812-819.

77. Connolly AJ, Ishihara H, Kahn ML, Farese RV, Coughlin SR. Role of the thrombin receptor in development and evidence for a second receptor. Nature. 1996;381(6582):516-519.

78. Luyendyk JP, Sullivan BP, Guo GL, Wang R. Tissue factor-deficiency and protease activated receptor-1-deficiency reduce inflammation elicited by diet-induced steatohepatitis in mice. $A m J$ Pathol. 2010;176(1):177-186.

79. Pfaffl MW. A new mathematical model for relative quantification in real-time RT-PCR. Nucleic Acids Res. 2001;29(9):e45.

80. Kopec AK, et al. Fibrin(ogen) drives repair after acetaminophen-induced liver injury via leukocyte $\alpha \mathrm{M} \beta 2$ integrin-dependent upregulation of Mmp12. J Hepatol. 2017;66(4):787-797. 